\title{
Oxidation/Reduction Studies on Nanoporous Platinum Films by Electrical Resistance
}

\section{Measurements}

\author{
Liangzhu Zhu ${ }^{1}$, Siddharth Kapoor ${ }^{2}$, Quintin Parry ${ }^{1}$, Ajay Nahata ${ }^{2}$, and Anil V. Virkar ${ }^{1, *}$ \\ ${ }^{1}$ Department of Materials Science and Engineering \\ ${ }^{2}$ Department of Electrical and Computer Engineering \\ University of Utah \\ Salt Lake City, UT 84112 \\ *Contributing Author \\ Email: anil.virkar@utah.edu
}

\begin{abstract}
Mechanisms and kinetics of surface reactions in nanoporous platinum films were investigated. Nanoporous films of platinum of $\sim 250 \mathrm{~nm}$ thickness were deposited on glass slides by cosputtering Pt and carbon followed by subsequently burning off carbon in air at $450^{\circ} \mathrm{C}$. Electrical resistance was measured in air and in $10 \% \mathrm{H}_{2}+$ nitrogen at $80^{\circ} \mathrm{C}$ as a function of time. The change in electrical resistance was extremely fast when switched to the $\mathrm{H}_{2}+\mathrm{N}_{2}$ atmosphere. When switched to air, the film resistance increased with time at a much slower rate. The increase
\end{abstract}


in resistance in air was attributed to the formation of Pt-oxide on the internal surfaces of the nanoporous films. The kinetics of oxidation was described by a model which includes two surface kinetic steps and a diffusional step. The use of nanoporous films makes it possible to investigate mechanisms and kinetics of surface reactions by ensuring a large surface to volume ratio. Oxide scale thickness at $80^{\circ} \mathrm{C}$ in air after several hours of oxidation was only submonolayer. Oxide scale thickness after $3 \mathrm{~h}$ at $450^{\circ} \mathrm{C}$ was about $1 \mathrm{~nm}$. Implications of the results for proton exchange membrane fuel cell (PEMFC) Pt catalyst degradation are discussed.

Key Words: Pt oxidation, nanoporous films, PEMFC Cathode Degradation

\section{Introduction}

Platinum (Pt) is one of the most widely used catalysts for numerous chemical and electrochemical processes. Nanosize Pt particles supported on high surface area carbon are used as both cathode and anode in proton exchange membrane fuel cells (PEMFC) [1]. To ensure a high catalytic activity, it is necessary that Pt catalyst exhibit a high surface area. Typical particle size of $\mathrm{Pt}$ in PEMFC electrodes is 2 to $5 \mathrm{~nm}$. The high surface area necessary to ensure reasonable reaction rates also promotes other competing (often deleterious) reactions. These include for instance chemisorption of $\mathrm{CO}$ at the anode and oxidation of Pt (especially at high voltages) at the cathode. An oxidative atmosphere at the cathode can lead to Pt oxidation and dissolution into the aqueous medium and in the monomer [2-6]. This leads to two unwanted reactions: (a) Growth of Pt particles by dissolution/re-precipitation, thus lowering the catalytic 
activity [4]. (b) Dissolution of $\mathrm{Pt}$ at the cathode, transport into the membrane as $\mathrm{Pt}^{2+}$ ions, and precipitation of Pt inside the membrane [6]. This latter phenomenon not only lowers the catalytic activity (due to the loss of Pt from the cathode) but also causes membrane degradation. It is thus of great importance, both scientifically and technologically, to investigate the interaction of Pt with other species with which chemical reactions are expected. The two of greatest interest include: (a) Reaction with CO, which can drastically lower anode activity. (b) Reaction with oxygen which can result in the oxidation of Pt at the cathode. In PEMFC, the oxidized Pt can dissolve into the liquid and may participate in other unwanted reactions. A study of the interaction of $\mathrm{Pt}$ with oxygen in the absence of liquid water is of interest as it can potentially isolate the role of oxygen in catalyst degradation. Such results are also of interest in high temperature PEMFC which may operate at temperatures $>100^{\circ} \mathrm{C}$ such that no liquid water is present. Transport of ionized Pt, however, can occur through the ionomer and is expected to affect catalyst durability even in the absence of water.

One of the approaches to investigate the interaction of Pt with various gaseous species involves the study some property that changes as a chemical reaction or an interaction occurs with the gaseous atmosphere. One such property is the electrical conductance (or resistance) measured as a function of time. Any adsorption of gases on the surface of Pt or reaction to form another phase is expected to change the total electrical resistance. For example, Berry [7] investigated the oxidation of Pt wires by measuring the electrical resistance as a function of time in oxygencontaining atmospheres. Such an approach may also be used with dense Pt films deposited on some support, such as glass slides. If the film is relatively thick the reaction is limited only to the 
surface and the resulting change in electrical resistance is expected to be rather modest similar to the observations of Berry on platinum wires [7].

In the present work we investigated the kinetics of oxidation/reduction using nanoporous $\mathrm{Pt}$ films. The use of nanoporous films vastly increases the total surface area exposed to oxidizing/reducing environments compared to dense films thus increasing the net signal (change in electrical resistance). Also, the use of nanoporous films should drastically increase the kinetics thus facilitating studies of oxidation/reduction at relatively low temperatures in a reasonable period of time. By contrast, when bulk samples (wires) are used, experiments need to be conducted for weeks or months to observe any detectable response [7].

Nanoporous films of Pt of $\sim 250 \mathrm{~nm}$ thickness were deposited on glass slides by sputtering using Pt and carbon targets. Porosity was formed in the films after burning off carbon in air. The electrical resistance of the film was measured as a function of time at $80^{\circ} \mathrm{C}$ in oxidizing and reducing environments. All measurements were conducted under no applied voltage. A theoretical model was developed to describe the kinetics of oxidation.

\section{Experimental Procedure}

Design and Construction of the Test System: The test chamber consisted of a fused silica glass cylinder clamped between two metallic plates with O-rings to ensure gas-tight seals. An 
electrical heater and a sample holder were inserted into the chamber. A thermocouple was introduced into the chamber to measure the sample temperature. Electrical feed-throughs were inserted from the end plates into the chamber. Also connected to the end plates were gas inlet/outlet fittings. The chamber was connected to a vacuum pump. A gas supply system consisting of flow meters and valves was used to introduce various atmospheres into the chamber. Electrical measurements were conducted using Keithley Model 2000 meters. Data acquisition was done using LabView software. Figure 1 shows a schematic and a photograph of the testing stand.

Deposition of Nanoporous Platinum Films on Glass Slides: For the formation of nanoporous Pt films, Pt and carbon were co-deposited using the two targets by RF and DC sputtering, respectively. The base chamber pressure was $2.4 \times 10^{-6}$ Torr. Pt was sputtered using $50 \mathrm{~W}$ of RF power, while carbon was deposited using $300 \mathrm{~W}$ of power. After sputtering, the samples were heated in air at $450^{\circ} \mathrm{C}$ for $3 \mathrm{~h}$ to burn off the deposited carbon and form platinum films with nanostructured porosity.

Measurement of Electrical Resistance in Various Atmospheres: The sample was inserted into the test chamber with electrical leads connected for a two-probe measurement, maintaining a distance of $8.5 \mathrm{~mm}$ between the probes. All measurements were conducted under no applied voltage. Initially, power to the heater was turned on, and the vacuum pump was turned on to evacuate the chamber to an end pressure of $7.5 \times 10^{-3}$ Torr. The sample temperature and the electrical resistance of the sample were continuously recorded. After the temperature reached $80^{\circ} \mathrm{C}$, both the temperature and the resistance were recorded for several hours. An evacuation 
step in between changes of atmosphere ensured an almost instantaneous change of atmosphere inside the chamber. Direct change of atmosphere without an evacuation step was too sluggish, as to be expected, since such a situation resembles a continuously stirred tank reactor. A gas mixture containing $10 \% \mathrm{H}_{2}+\mathrm{N}_{2}$ was introduced into the chamber to reduce any platinum oxide(s) that may have formed during carbon burn off in air. After a short exposure to an $\mathrm{H}_{2}$ containing atmosphere, all of the inlet and the outlet valves were closed once again and the chamber was evacuated. After achieving a vacuum of $7.5 \times 10^{-3}$ Torr, flowing air was again introduced into the chamber and the electrical resistance of the sample was continuously measured for several hours. This procedure was repeated several times.

Characterization of the Nanoporous Platinum Films: The as-formed Pt films and after exposure to various atmospheres were characterized by X-ray diffraction (XRD), scanning electron microscopy (SEM), X-ray photoelectron spectroscopy (XPS) and high resolution transmission electron microscopy (HRTEM). The volume fraction of platinum $\left(V_{v}\right)$ and the pore surface area per unit volume $\left(S_{v}\right)$ were measured on SEM images using quantitative stereology [8]. The volume fraction of Pt was measured using the systematic point count method, where $V_{v}=P_{P t}$ in which $P_{P t}$ is the fraction of the grid points falling on Pt on the SEM images. The pore surface area per unit volume is given by $S_{v}=2 P_{L}$ where $P_{L}$ denotes the number of intersections of Pt/pore boundaries with a test line applied to the SEM images [8]. 


\section{Results and Discussion}

Microstructure of the Nanoporous Pt Film: Figure 2 shows an SEM image of the nanoporous Pt film formed by co-sputtering Pt and C, followed by burning off carbon at $450^{\circ} \mathrm{C}$ in air for $3 \mathrm{~h}$. The micrograph shows that the individual particle size (feature) is on the order of a few nanometers and the pores are also on the order of a few nm in size. Thus, when exposed to various gaseous atmospheres, we expect rapid gaseous transport to occur into and/or out of the pore space of the film. An approximate estimate of the time needed for change of atmosphere to occur through the film of thickness $l$ is given by $t \sim \frac{l^{2} \tau}{D_{K} V_{p}}$ where $D_{K}=\frac{1}{3} \sqrt{\frac{8 k_{B} T}{\pi m}} d_{p}$ is the Knudsen diffusivity, $d_{p}$ is the pore diameter, $m$ is the molecular mass of the diffusing gas, $V_{p}$ is the volume fraction of porosity, and $\tau$ is the tortuosity factor. For $d_{p}=10 \mathrm{~nm}$, assuming the diffusing gas to be oxygen (molecular mass of $32 / N_{A}$ g where $N_{A}$ is the Avogadro number), the $D_{K} \approx 0.016 \mathrm{~cm}^{2} \mathrm{~s}^{-1}$ at $80^{\circ} \mathrm{C}$. Assuming $V_{p}=0.4$, a tortuosity factor of 3 [9], for $l=250 \mathrm{~nm}$, the time needed for the change of atmosphere to occur through the thickness of the film is on the order of $0.3 \mu \mathrm{s}$. Indeed, extremely rapid exchange of atmosphere through the thickness of the film is expected. Thus, the slower step is actually the exchange of gaseous atmosphere inside the chamber upon switching of gases. It is for this reason the chamber was evacuated between changes of atmospheres. 
General Observations of Temperature Variation during Testing: All of the testing in this study was conducted at nominally $80^{\circ} \mathrm{C}$. The electrical heater used to heat the sample and the surrounding atmosphere were controlled by an on/off controller. Figure 3 shows the temperature vs. time trace during a run in which the sample was subjected to multiple atmospheres including vacuum. Various observations made and their origin are briefly described and discussed in what follows.

Figure 3 shows the measured electrical resistance and temperature as the sample was heated in vacuum from room temperature to $80^{\circ} \mathrm{C}$. This sample was initially heated in air at $450^{\circ} \mathrm{C}$ for $3 \mathrm{~h}$ after sputter deposition to burn off carbon prior to its introduction into the test chamber. Thus, the sample is expected to have some oxide scale on the pore surfaces of the nanoporous film. Note that as the temperature was increased, the sample resistance also increased in a manner consistent with the temperature dependence of the electrical resistivity of metallic platinum. The inset shows the dependence of resistance on temperature. After the temperature reached the nominal set point of $80^{\circ} \mathrm{C}$, the temperature began to oscillate. The power controller used was on/off type. The thermal inertia of the sample and the surroundings in vacuum is presumably low resulting in temperature oscillations and corresponding oscillations in the measured resistance of the Pt film. The approximate value of the resistance was $\sim 56.25 \Omega$ after the temperature reached the set point of $80^{\circ} \mathrm{C}$, but thereafter slightly decreased to $\sim 55.5 \Omega$.

Subsequently, a gaseous mixture of $10 \% \mathrm{H}_{2}+\mathrm{N}_{2}$ was introduced into the chamber. The corresponding resistance vs. time and temperature vs. time plots are shown in Figure 4. As soon as $10 \% \mathrm{H}_{2}+\mathrm{N}_{2}$ atmosphere was introduced, the sample resistance dropped sharply to about 28 
$\Omega$ and remained nearly constant thereafter. We attribute the large drop in resistance after hydrogen was introduced to the reduction of any oxide scale formed during the earlier carbon burn off step. The temperature rose sharply by about $10^{\circ} \mathrm{C}$, and then returned to the set point of $80^{\circ} \mathrm{C}$. The sharp rise in temperature is attributed to the reduction of platinum oxide to platinum. Assuming the existence of some $\mathrm{PtO}_{2}$ on the surface of $\mathrm{Pt}$, the expected reaction upon the introduction of hydrogen is

$$
\mathrm{PtO}_{2}+2 \mathrm{H}_{2} \rightarrow \mathrm{Pt}+2 \mathrm{H}_{2} \mathrm{O}
$$

This is a highly exothermic reaction with a molar enthalpy of $-390 \mathrm{~kJ} \cdot \mathrm{mol}^{-1}[10,11]$. The kinetics of reduction is expected to be fast, as observed. This is because as $\mathrm{PtO}_{2}$ is reduced to $\mathrm{Pt}$, there is a volume decrease since the molar volume of $\mathrm{PtO}_{2}\left(22.26 \mathrm{ml}^{\left.-\mathrm{mol}^{-1}\right)}\right.$ is much higher than the molar volume of Pt $\left(9.048 \mathrm{ml} . \mathrm{mol}^{-1}\right)$. Because of this volume change, tiny, nanometer scale cracks and fissures are expected to form through which gas transport occurs fast and the kinetics of reduction are expected to be interface-controlled. The heat is generated rapidly, but is not dissipated easily thus causing a sharp increase in temperature. It is further observed that once the temperature returns to the set point of $80^{\circ} \mathrm{C}$, the oscillations all but disappear. The absence of oscillations is attributed to the increased thermal inertia of the system in the presence of the flowing gas. Effectively, convective heat transfer that occurs in the gas phase evens out the temperature and the oscillations disappear (or become imperceptibly small).

At time $\mathrm{t} 2$ (Figure 4), the atmosphere was again changed to vacuum. The film resistance essentially remained unchanged. There was again a sudden rise in temperature. This rise in 
temperature is attributed to a sudden decrease in the thermal inertia of the sample + surroundings as the chamber is evacuated (the near absence of a gas phase). The power controller is relatively slow to respond. Thus, it continues to put out power at a greater rate (as would be required to heat up the gas phase while the gas phase is present) than needed for the sample to be maintained at the set point under vacuum. Sudden removal of the gas phase thus lowers the thermal inertia and in response the temperature goes up. The power controller adjusts to the new environment (vacuum) in time, lowers the power output and the temperature drops down. For the same reasons as stated earlier, the oscillations reappear and continue as long as the atmosphere in the chamber is vacuum.

At time $t_{3}$, air was introduced into the chamber. Plots of the electrical resistance and temperature vs. time are shown in Figure 5. A sharp rise in temperature was observed, which lasted for a very short duration. This is attributed to some surface oxidation of $\mathrm{Pt}$ to $\mathrm{PtO}_{2}$, since the reaction

$$
\mathrm{Pt}+\mathrm{O}_{2} \rightarrow \mathrm{PtO}_{2}
$$

is also exothermic with a molar enthalpy of $-100 \mathrm{~kJ} \cdot \mathrm{mol}^{-1}[10]$. The observed sharp spike in temperature suggests that the reaction was primarily restricted to the surface. Thereafter the temperature remains constant at the set point of $80^{\circ} \mathrm{C}$ with no oscillations. The electrical resistance continued to increase from an initial value of $\sim 27 \Omega$ to about $\sim 30.5 \Omega$ over a period of about $18 \mathrm{~h}$. By contrast, because of the use of thick, bulk wires in the work of Berry [7] the change in resistance after several days was only in the milliohms range. The increase in resistance in the present work (Figure 5) was relatively rapid in the beginning but then the rate of 
increase continuously decreased. Subsequent switches in atmosphere to vacuum and later to $10 \%$ $\mathrm{H}_{2}+\mathrm{N}_{2}$ and to air essentially reproduced the observations described in the foregoing.

After a similar test (oxidation) on another sample, the sample was removed from the test chamber and was examined by XRD. No oxide scale was detected. This is not surprising as the $\mathrm{XRD}$ technique is not very sensitive if the oxide scale is very thin (less than a few nanometers). Figure 6 shows XPS spectra of the sample after various treatments. (a) XPS spectra of the as deposited Pt + carbon film: Pt4d and Pt4f peaks, carbon 1s peak and a small peak corresponding to O1s are observed. This small O1s peak is attributed to the expected adsorption of oxygen on the surface of Pt when exposed to air in a two phase Pt $+\mathrm{C}$ mixture. (b) XPS spectra of the film after burning off carbon at $450^{\circ} \mathrm{C}$ for $3 \mathrm{~h}$ : A strong O1s peak is observed with weak Pt4d and Pt $4 \mathrm{f}$ peaks, and a weak C1s peak. A strong O1s is consistent with the expectation that surface oxide formed on the pore surface when heated in air. (c) XPS spectra of the film after exposure to hydrogen-containing atmosphere at $80^{\circ} \mathrm{C}$ : A strong O1s peak is still observed in addition to the Pt peaks. The sample after hydrogen treatment at $80^{\circ} \mathrm{C}$ was exposed to air for a short period of time before placing it in the XPS chamber. The XPS spectra show that even a brief exposure to air at room temperature is sufficient to form a layer of oxygen (possibly chemisorbed). The oxygen partial pressure, $p_{O_{2}}$, for $\mathrm{Pt} / \mathrm{PtO}_{2}$ equilibrium at room temperature, based on the available thermodynamic data $[10,11]$, is estimated to be only $\sim 4.4 \times 10^{-16}$ atm. Thus, either surface oxidation or chemisorption of oxygen is readily expected to occur upon exposure to air. (d) XPS spectra after exposure to air at $80^{\circ} \mathrm{C}$ : Again a strong O1s peak is observed. The Pt peaks are slightly weaker than in the sample exposed to hydrogen (c) as to be expected. Peaks corresponding to $\mathrm{Na}$ and $\mathrm{Si}$ are attributed to some contamination in sample handling. 
Model for Oxidation: We consider a porous Pt film with $V_{v}$ as the volume fraction of Pt (so the porosity is given by $V_{p}=1-V_{v}$ ), pore surface area of the film per unit volume given by $S_{v}$ (in $\mathrm{cm}^{2} \cdot \mathrm{cm}^{-3}$ or $\mathrm{cm}^{-1}$ ), length $\ell$ (between the probes for the measurement of electrical resistance), and cross-sectional area of $A$. We will assume that the oxide formed is of the stoichiometry $\mathrm{PtO}_{2}$ [12]. We will also assume that the approximate particle size of $\mathrm{Pt}$ in the nanoporous film is much larger than the thickness of the oxide film formed, $\delta(t)$, at any time $t$ over the duration of the experiments. This is in accord with several reported studies which show that a measureable oxide scale is not observed under similar conditions (e.g. $80^{\circ} \mathrm{C}$ ). Figure 7 shows a schematic of a nanoporous $\mathrm{Pt}$ structure with a thin layer of oxide, $\delta(t)$, covering the pore surface. Let the electrical resistivities of $\mathrm{Pt}$ and $\mathrm{PtO}_{2}$ be $\rho_{P t}$ and $\rho_{P t O_{2}}$, respectively, in $\Omega \mathrm{cm}$. At time $t=0$, the sample resistance is given by

$$
R_{o}=\frac{\rho_{P t} \ell}{A V_{v}}
$$

At some time $t=t$, the inverse sample resistance is given by

$$
\frac{1}{R(t)}=\frac{1}{R_{P t}(t)}+\frac{1}{R_{P t O_{2}}(t)}=\frac{1}{\frac{\rho_{P t} \ell}{A\left(V_{v}-S_{v} \delta(t)\right)}}+\frac{1}{\frac{\rho_{P t O_{2}} \ell}{A S_{v} \delta(t)}}
$$

This assumes that the two resistances are in parallel. Thus,

$$
R(t)=\frac{R_{P t}(t) R_{P_{t O} O_{2}}(t)}{R_{P t}(t)+R_{P t O_{2}}(t)}
$$

Equation (2) for the inverse resistance can also be written as

$$
\frac{1}{R(t)}=\frac{A V_{v}}{\ell \rho_{P t}}-\frac{A S_{v}\left(\rho_{P t O_{2}}-\rho_{P t}\right)}{\ell \rho_{P t} \rho_{P t O_{2}}} \delta(t)=\frac{1}{R_{O}}-\frac{A S_{v}\left(\rho_{P t O_{2}}-\rho_{P t}\right)}{\ell \rho_{P t} \rho_{P t O_{2}}} \delta(t)
$$


We now consider the kinetics of oxidation. There are two interface steps; one at the gas phase $/ \mathrm{PtO}_{2}$ interface and the other at the $\mathrm{Pt} / \mathrm{PtO}_{2}$ interface. At the gas phase $/ \mathrm{PtO}_{2}$ interface, possible sub-steps include: (1) Adsorption of $\mathrm{O}_{2}$ on $\mathrm{PtO}_{2}: \mathrm{O}_{2}$ (gas) $\rightarrow \mathrm{O}_{2}$ (ads). (2) Dissociation of $\mathrm{O}_{2}$ adsorbed into $\mathrm{O}$ adsorbed: $\mathrm{O}_{2}(\mathrm{ads}) \rightarrow 2 \mathrm{O}$ (ads). (3) Incorporation of the adsorbed $\mathrm{O}$ into the $\mathrm{PtO}_{2}$ lattice: $\mathrm{O}($ ads $) \rightarrow \mathrm{O}\left(\right.$ in $\left.\mathrm{PtO}_{2}\right)$. The overall kinetics may be described by a kinetic factor $k^{\prime}$ (expected to be thermally activated, but containing many factors).

Diffusion of $\mathrm{O}$ occurs through the $\mathrm{PtO}_{2}$ film of thickness $\delta(t)$. There are many possible mechanisms. This diffusion will likely occur by some defect mechanism (vacancies, and electrons and/or holes). The net transport may still be described by the diffusion of $\mathrm{O}_{2}$ given by $D_{\mathrm{O}_{2}}$ (expected to be thermally activated). The actual transport may occur as diffusion of O (e.g. $\left.D_{O}\right)$, such as by a coupled transport of $\mathrm{O}^{2-}$ ions via oxygen vacancies, $V_{O}^{\bullet \bullet}$, and electrons [13].

At the $\mathrm{PtO}_{2} / \mathrm{Pt}$ interface, the reaction involves $2 \mathrm{O}+\mathrm{Pt} \rightarrow \mathrm{PtO}_{2}$. The corresponding kinetic factor (also expected to be thermally activated) is given by $k^{\prime \prime}$. We consider a quasi-steady state oxidation kinetics. The oxygen chemical potential in the gas phase is given by $\mu_{\mathrm{O}_{2}}^{I}$. The oxygen chemical potential in $\mathrm{Pt}$ (corresponding to whatever may be the small amount of dissolved oxygen in $\mathrm{Pt}$ ) is given by $\mu_{\mathrm{O}_{2}}^{I I}$. This is the one corresponding to the reaction equilibrium $\mathrm{Pt}+\mathrm{O}_{2}$ $\rightarrow \mathrm{PtO}_{2}$ in which $\mathrm{Pt}$ and $\mathrm{PtO}_{2}$ are assumed to be in their standard states (pure). There will be sharp changes in the chemical potentials of oxygen at the gas phase $/ \mathrm{PtO}_{2}$ interface and at the $\mathrm{PtO}_{2} / \mathrm{Pt}$ interface. Let $\mu_{O_{2}}^{\prime}$ be the chemical potential of oxygen in the $\mathrm{PtO}_{2}$ film close to the gas phase $/ \mathrm{PtO}_{2}$ interface. Let $\mu_{O_{2}}^{\prime \prime}$ be the chemical potential of oxygen in the $\mathrm{PtO}_{2}$ film close to the 
$\mathrm{PtO}_{2} / \mathrm{Pt}$ interface. Figure 8 shows a schematic of the variation of the chemical potential of oxygen, $\mu_{O_{2}}$, through the film and across the interfaces.

In a quasi-steady state, the oxygen flux, $j_{O_{2}}$, is given by

$$
j_{O_{2}}=k^{\prime}\left(\mu_{O_{2}}^{I}-\mu_{O_{2}}^{\prime}\right)=\frac{D_{O_{2}} C_{O_{2}}}{k_{B} T}\left(\frac{\mu_{O_{2}}^{\prime}-\mu_{O_{2}}^{\prime \prime}}{\delta(t)}\right)=k^{\prime \prime}\left(\mu_{O_{2}}^{\prime \prime}-\mu_{O_{2}}^{I I}\right)=\frac{1}{V_{M}} \frac{d \delta(t)}{d t}
$$

In equations (5) $V_{M}$ is the molecular volume of $\mathrm{PtO}_{2}$. The chemical potentials of oxygen in the $\mathrm{PtO}_{2}$ oxide film adjacent to the gas phase/PtO ${ }_{2}$ interface $\left(\mu_{O_{2}}^{\prime}\right)$ and adjacent to the $\mathrm{PtO} 2 / \mathrm{Pt}$ interface $\left(\mu_{O_{2}}^{\prime \prime}\right)$ are not easily measureable. These, however, can be eliminated by solving the set of equations (5).

The preceding assumes that the chemical potential of oxygen, $\mu_{O_{2}}$, varies linearly through the oxide film and that $D_{O_{2}}$ and $C_{O_{2}}$ represent average values. In general, the variation of $\mu_{O_{2}}$ need not be linear and it is possible to include position dependent values if known, in which case the flux of oxygen through the film is given by

$$
j_{O_{2}}=\frac{1}{k_{B} T \delta(t)} \int_{\mu_{O_{2}}^{\prime}}^{\mu_{O_{2}}^{\prime \prime}} D_{O_{2}}(x) C_{O_{2}}(x)\left(-d \mu_{O_{2}}(x)\right)
$$

In most experimental studies, it is not possible to obtain position dependent values of parameters, and what is reflected in most measurements, e.g. oxidation kinetics, are the effects of average values, namely $D_{O_{2}}$ and $C_{O_{2}}$. It is for this reason in the following analysis, average values of $D_{\mathrm{O}_{2}}$ and $C_{\mathrm{O}_{2}}$ for the oxide film are used and the analysis assumes a linear variation of $\mu_{\mathrm{O}_{2}}$ through the film. 
Solution to the set of equations (5) (upon integration) gives

$$
\frac{\delta(t)^{2}}{2 V_{M}}+\frac{D_{O_{2}} C_{O_{2}}}{V_{M} k_{B} T}\left(\frac{k^{\prime}+k^{\prime \prime}}{k^{\prime} k^{\prime \prime}}\right) \delta(t)=\frac{D_{O_{2}} C_{O_{2}}}{k_{B} T}\left(\mu_{O_{2}}^{I}-\mu_{O_{2}}^{I I}\right) t
$$

which is a quadratic equation in $\delta(t)$ as a function of time $t$. Equation (7), which describes the time dependence of the oxide scale in terms of interface kinetics and bulk transport through the film, is essentially the well-known Wagner model for oxidation (including interface reactions) [13].

We now consider two limiting cases.

Suppose

$$
\frac{\delta(t)^{2}}{2 V_{M}}<<\frac{D_{O_{2}} C_{O_{2}}}{V_{M} k_{B} T}\left(\frac{k^{\prime}+k^{\prime \prime}}{k^{\prime} k^{\prime \prime}}\right) \delta(t)
$$

(at short times - early stage, that is small $\delta(t)$ )

This gives

$$
\delta(t) \approx \frac{V_{M} k^{\prime} k^{\prime \prime}}{\left(k^{\prime}+k^{\prime \prime}\right)}\left(\mu_{O_{2}}^{I}-\mu_{O_{2}}^{I I}\right) t
$$

or

$$
\delta(t) \approx k_{e f f} t
$$

where we have written

$$
k_{e f f} \approx \frac{V_{M} k^{\prime} k^{\prime \prime}}{\left(k^{\prime}+k^{\prime \prime}\right)}\left(\mu_{O_{2}}^{I}-\mu_{O_{2}}^{I I}\right)
$$

as the effective interface reaction parameter. Note that the smaller of the two interface kinetic parameters, $k^{\prime}$ or $k^{\prime \prime}$, controls the interface kinetics as the two are series steps. 
Thus, initially the time dependence of the oxide thickness, $\delta(t)$, is linear.

Now suppose

$$
\frac{\delta(t)^{2}}{2 V_{M}}>>\frac{D_{O_{2}} C_{O_{2}}}{V_{M} k_{B} T}\left(\frac{k^{\prime}+k^{\prime \prime}}{k^{\prime} k^{\prime \prime}}\right) \delta(t)
$$

(at a later stage, that is relatively large $\delta(t)$ )

Then we have

$$
\delta(t)^{2} \approx \frac{2 V_{M} D_{O_{2}} C_{O_{2}}}{k_{B} T}\left(\mu_{O_{2}}^{I}-\mu_{O_{2}}^{I I}\right) t
$$

or

$$
\delta(t)^{2} \approx D_{e f f} t
$$

This is essentially the Wagner parabolic law of oxidation [13]. In the foregoing, it has been assumed that oxidation predominantly involves transport on the anion sublattice. The nature of the equation, however, remains essentially the same even in the case transport predominantly occurs on the cation sublattice. In the above, we have written

$$
D_{e f f}=\frac{2 V_{M} D_{O_{2}} C_{O_{2}}}{k_{B} T}\left(\mu_{O_{2}}^{I}-\mu_{O_{2}}^{I I}\right)
$$

as the effective diffusion coefficient.

Thus,

$$
\delta(t) \approx \sqrt{D_{e f f} t}
$$


Using the above equations, we now explore the time dependence of the inverse resistance. At short times, we expect

$$
\frac{1}{R(t)}=\frac{A V_{v}}{\ell \rho_{P t}}-\frac{A S_{v}}{\ell}\left(\frac{\rho_{P t O_{2}}-\rho_{P t}}{\rho_{P t O_{2}} \rho_{P t}}\right) \delta(t) \approx \frac{A V_{v}}{\ell \rho_{P t}}-\frac{A S_{v}}{\ell}\left(\frac{\rho_{P t O_{2}}-\rho_{P t}}{\rho_{P t O_{2}} \rho_{P t}}\right) k_{e f f} t
$$

That is, at short times we expect a plot of $\frac{1}{R(t)}$ vs. $t$ to be linear with $\frac{A V_{v}}{\ell \rho_{P t}}$ as the intercept and $-\frac{A S_{v}}{\ell}\left(\frac{\rho_{P t O_{2}}-\rho_{P t}}{\rho_{P t O_{2}} \rho_{P t}}\right) k_{e f f}$ as the slope. If the resistivity of $\mathrm{PtO}_{2}$ is much greater than that of $\mathrm{Pt}$, that is if $\rho_{P t O_{2}}>\rho_{P t}$, the slope is given by $-\frac{A S_{v}}{\ell \rho_{P t}} k_{e f f}$. Indeed, results of Abe et al. [12] and others [7] have shown that $\rho_{P_{t} O_{2}}>\rho_{P t}$. Thus, the magnitude of the ratio of the slope to the intercept at short times gives $k_{\text {eff }}$; that is

$$
\frac{\mid \text { Slope } \mid}{\mid \text { Intercept }}=\frac{\left|-\frac{A S_{v}}{\ell \rho_{P t}} k_{e f f}\right|}{\left|\frac{A V_{v}}{\ell \rho_{P t}}\right|}=\frac{S_{v}}{V_{v}} k_{e f f}
$$

At long times, we expect

$$
\frac{1}{R(t)}=\frac{A V_{v}}{\ell \rho_{P t}}-\frac{A S_{v}}{\ell}\left(\frac{\rho_{P t O_{2}}-\rho_{P t}}{\rho_{P t O_{2}} \rho_{P t}}\right) \delta(t) \approx \frac{A V_{v}}{\ell \rho_{P t}}-\frac{A S_{v}}{\ell}\left(\frac{\rho_{P t O_{2}}-\rho_{P t}}{\rho_{P t O_{2}} \rho_{P t}}\right) \sqrt{D_{e f f}} \sqrt{t}
$$

Thus, at long times we expect a plot of $\frac{1}{R(t)}$ vs. $\sqrt{t}$ to be linear, with the intercept again given by $\frac{A V_{v}}{\ell \rho_{P t}}$ but the slope given by $-\frac{A S_{v}}{\ell}\left(\frac{\rho_{P t O_{2}}-\rho_{P t}}{\rho_{P t O_{2}} \rho_{P t}}\right) \sqrt{D_{e f f}}$. Since $\rho_{P t O_{2}}>>\rho_{P t}$, the slope is equal to $-\frac{A S_{v}}{\ell \rho_{P t}} \sqrt{D_{e f f}}$. Then, square of the ratio of the slope to the intercept gives $D_{e f f}$; that is 


$$
\frac{\mid \text { Slope }\left.\right|^{2}}{\mid \text { Intercept }\left.\right|^{2}}=\frac{\left|-\frac{A S_{v}}{\ell \rho_{P t}} \sqrt{D_{e f f}}\right|^{2}}{\left|\frac{A V_{v}}{\ell \rho_{P t}}\right|^{2}}=\left(\frac{S_{v}}{V_{v}}\right)^{2} D_{\text {eff }}
$$

Analysis of the Experimental Results: Figure 9 and Figure 10 respectively show the plots of $\frac{1}{R(t)}$ vs. $t$ and $\frac{1}{R(t)}$ vs. $\sqrt{t}$. The slope of $\frac{1}{R(t)}$ vs. $t$ as $t \rightarrow 0$ is $=-3.01 \times 10^{-6} \Omega^{-1} \mathrm{~s}^{-1}$ and the intercept is $=0.0367 \Omega^{-1}$. The slope of $\frac{1}{R(t)}$ vs. $\sqrt{t}$ at long times is $=-3.98 \times 10^{-6} \Omega^{-1} \mathrm{~s}^{-1 / 2}$ and the intercept is $=0.0335 \Omega^{-1}$. Using quantitative stereology [8] on the SEM micrographs, $V_{v}$ was found to be 0.6 and $S_{v}$ was found to be $2.34 \times 10^{6} \mathrm{~cm}^{-1}$.

Note that (Figure 10), at long times, the $\frac{\mid \text { Slope }\left.\right|^{2}}{\mid \text { Intercep }\left.\right|^{2}}=\frac{\left|-\frac{A S_{v}}{\ell \rho_{P t}} \sqrt{D_{e f f}}\right|^{2}}{\left|\frac{A V_{v}}{\ell \rho_{P t}}\right|^{2}}=\left(\frac{S_{v}}{V_{v}}\right)^{2} D_{\text {eff }}=(-3.98)^{2} \times 10^{-12} \mathrm{~s}^{-1}$.

The estimated effective diffusion coefficient is thus $D_{\text {eff }} \sim 1.0 \times 10^{-24} \mathrm{~cm}^{2} \mathrm{~s}^{-1}$. This is a very low value and practically immeasurable using gravimetry or oxide scale thickness measurement or other techniques. The maximum duration of time at $80^{\circ} \mathrm{C}$ the sample was exposed to air was $\sim 6.5$ $\mathrm{x} 10^{4} \mathrm{~s}$. Thus, the oxide scale thickness is estimated to be $2.55 \times 10^{-10} \mathrm{~cm}$ (sub-monolayer), and thus obviously not a continuous film. From the slope of $\frac{1}{R(t)}$ vs $t$ as $t \rightarrow 0$, the effective interface rate constant is estimated as $k_{\text {eff }} \sim 7.72 \times 10^{-13} \mathrm{~cm} . \mathrm{s}^{-1}$. Assuming this initial stage to last 
may be about $100 \mathrm{~s}$, the corresponding oxide scale thickness in the early stages would be $\sim 7.72 \mathrm{x}$ $10^{-11} \mathrm{~cm}$. Again, this is an exceptionally small value (sub-monolayer).

Both of these estimates amount to a fraction of an angstrom as the oxide scale thickness. This means over the duration of the experiment very thin oxide scale is formed, and the scale is not contiguous. In all likelihood, the oxide scale formed may only be at defects such as crevices or inter-particle necks. The very low value of the diffusivity estimated may, however, be quite reasonable as will be discussed later. In many of the refractory oxides, measurements of diffusion coefficient can realistically be done only at rather high temperatures, and the extrapolated values at low temperatures are exceptionally low (and practically immeasurable). As an example, measurements of the oxygen diffusion coefficient through $\mathrm{ZnO}$ are available in the literature [14]. The values range from $2.73 \times 10^{-17} \mathrm{~cm}^{2} \mathrm{~s}^{-1}$ at $850^{\circ} \mathrm{C}$ to $3.5 \times 10^{-14} \mathrm{~cm}^{2} \mathrm{~s}^{-1}$ at $1200^{\circ} \mathrm{C}$. Extrapolated value of the diffusivity at $80^{\circ} \mathrm{C}$ is $\sim 10^{-46} \mathrm{~cm}^{2} \mathrm{~s}^{-1}$, clearly not measureable (and not even a meaningful number in the context of a measurable parameter). No measurements are available on oxygen diffusion through $\mathrm{PtO}_{2}$. Nevertheless the effective diffusion coefficient of $\sim 1.0 \times 10^{-24} \mathrm{~cm}^{2} \mathrm{~s}^{-1}$ at $80^{\circ} \mathrm{C}$ estimated in the present work may be quite reasonable as discussed in what follows.

We first compare the present results with the prior work of Berry [7] on the oxidation of platinum wires and later also discuss the implications of the present work concerning the interaction of oxygen with platinum in proton exchange membrane fuel cells (PEMFC). Berry [7] investigated the oxidation kinetics of Pt wires over a range of temperatures between $450^{\circ} \mathrm{C}$ and $597^{\circ} \mathrm{C}$ for a total of $6000 \mathrm{~h}$. It was observed that $\mathrm{Pt}$ wires slowly oxidized over this 
temperature range. At $630^{\circ} \mathrm{C}$, however, platinum oxide rapidly reduced to metallic platinum, in accord with expectations based on the thermodynamics of platinum oxidation [10,11]. The oxide scale in one experiment of Berry grew to a thickness of $94 \mathrm{~nm}$. As the temperature was not constant in Berry's experiment, only an approximate estimate of $D_{\text {eff }}$ can be obtained assuming $\delta(t) \approx \sqrt{D_{\text {eff }} t}$. For $\delta(t) \approx 94 \mathrm{~nm}$ and time of $6000 \mathrm{~h}$, the estimated $D_{\text {eff }}$ from Berry's work is on the order of $4.0 \times 10^{-18} \mathrm{~cm}^{2} \mathrm{~s}^{-1}$. Since the minimum temperature in Berry's experiment was $450^{\circ} \mathrm{C}$, we will assume this to be the temperature at which $D_{\text {eff }} \sim 4.0 \times 10^{-18} \mathrm{~cm}^{2} \mathrm{~s}^{-1}$. In the present work, the estimated $D_{\text {eff }}$ at $80^{\circ} \mathrm{C}$ is $\sim 1.0 \times 10^{-24} \mathrm{~cm}^{2} \mathrm{~s}^{-1}$. Assuming a thermally activated behavior, we expect $D_{\text {eff }} \approx D_{o} \exp \left(-\frac{Q}{R T}\right)$ where $D_{o}$ is the pre-exponent and $Q$ is the activation energy. From these two measurements, we estimate an activation energy for the effective oxygen diffusion coefficient in $\mathrm{PtO}_{2}$ as $Q \sim 97 \mathrm{kJmol}^{-1}$. This is a very reasonable number when compared with other oxides [14].

Estimation of $D_{e f f}$ at $450^{\circ} \mathrm{C}$ from the Carbon Burn off Experiment: The nanoporous Pt films in the present work were made by co-sputtering Pt and carbon, and later by burning off carbon in air at $450^{\circ} \mathrm{C}$ for $3 \mathrm{~h}$. In this process, Pt oxidation on pore surfaces is expected. The initial resistance of $\sim 55.5 \Omega$ of the film in Figure 3 and Figure 4 (before exposure to hydrogen) corresponds to such a sample. After exposure to hydrogen, the sample resistance sharply dropped to $\sim 28 \Omega$ indicating reduction of the oxide scale to Pt. Thus, if we had a fully reduced sample (no oxide scale) as the starting sample in our oxidation studies, its resistance would have been $R_{o}=28 \Omega$. If the sample is then oxidized in air at $450^{\circ} \mathrm{C}$ for $3 \mathrm{~h}(10,800 \mathrm{~s})$, its resistance will be 
$R(t)=55.5 \Omega$ where $\mathrm{t}=10,800 \mathrm{~s}$. From this information, one can now determine $D_{\text {eff }}$ at $450^{\circ} \mathrm{C}$.

Note that equation (19) can be written as

$$
\frac{1}{R(t)}=\frac{1}{R_{o}}-\frac{1}{R_{o}} \frac{S_{v}}{V_{v}} \sqrt{D_{e f f} t}
$$

or the effective diffusion coefficient is given by

$$
D_{e f f}=\left(\frac{V_{v}}{S_{v}}\right)^{2} \frac{1}{t}\left(1-\frac{R_{O}}{R(t)}\right)^{2}
$$

While the carbon burn off was done at $450^{\circ} \mathrm{C}$, the resistances $\left(R_{o}\right.$ and $\left.R(t)\right)$ were measured at $80^{\circ} \mathrm{C}$. Note that the ratio $R_{o} / R(t)$ should be the same whether the electrical resistance measurements are made at $450^{\circ} \mathrm{C}$ or $80^{\circ} \mathrm{C}$ since $\rho_{P t O_{2}}>>\rho_{P t}$. From the present experimental data, the estimated value of $D_{\text {eff }}$ at $450^{\circ} \mathrm{C}$ is $D_{\text {eff }} \approx 1.495 \times 10^{-18} \mathrm{~cm}^{2} \mathrm{~s}^{-1}$. This result obtained from the measurement of the electrical resistance of nanoporous Pt films in the present work is in remarkable agreement with the $D_{\text {eff }}$ of $4.0 \times 10^{-18} \mathrm{~cm}^{2} \mathrm{~s}^{-1}$ estimated from oxide thickness measurement on bulk Pt wires used in Berry's work [7]. A slightly higher value of the $D_{\text {eff }}$ based on Berry's data is expected because temperature in those studies was varied between $450^{\circ} \mathrm{C}$ and $597^{\circ} \mathrm{C}$. Using the estimated value of $D_{e f f} \approx 1.495 \times 10^{-18} \mathrm{~cm}^{2} \mathrm{~s}^{-1}$ in the present work we estimate that the oxide scale thickness on the pore surfaces of the nanoporous Pt films after a $3 \mathrm{~h}$ treatment in air at $450^{\circ} \mathrm{C}$ to be $\delta(t) \approx \sqrt{D_{e f f} t}$ which turns out to be $\sim 1.27 \mathrm{~nm}$. Thus, the estimated oxide scale thickness after carbon burn off in the present work is $\sim 1.27 \mathrm{~nm}$.

Figure 11 is high resolution transmission electron micrograph (HRTEM) of the film after carbon burn off at $450^{\circ} \mathrm{C}$ in air. The central light region is a pore. The image shows that the film is 
polycrystalline with grain (particle) size of $\sim 5 \mathrm{~nm}$. The inset shows Fast Fourier transform (FFT) diffraction pattern. The diffraction rings are identified as corresponding to $\{111\}$ planes with a dspacing of $0.226 \mathrm{~nm}$ and $\{200\}$ planes with a d-spacing of $0.196 \mathrm{~nm}$. However, diffraction spots corresponding to $\mathrm{PtO}_{2}$ were not detected, possibly due to its very small size (thickness) and apparent disordered structure.

Figure 12 is a higher magnification image of the region surrounding the pore in Figure 11. Enlarged images of regions ' $a$ ' and ' $b$ ' are shown as insets, which clearly show the corresponding lattice images. Also evident in the insets are interplanar spacings of $0.23 \mathrm{~nm}$ corresponding to Pt $\{111\}$ planes. The regions adjacent to the pore marked 'c', 'd', and 'e' exhibit different morphology than the interior Pt grains. These regions appear to be disordered and their morphology is distinct from the Pt regions. The thickness of these regions is about 1 to $2 \mathrm{~nm}$. Because of their smaller size and low volume fraction, we are unable to definitely confirm that these regions are Pt-oxide. However, since O has atomic weight of 16 and Pt has 195, we expect Pt-oxide to appear lighter in the bright field image, as observed. Thus, it may be concluded that the HRTEM image is consistent with the formation of a Pt-oxide scale along the periphery of the pore (and thus along the peripheries of other pores) upon heat treatment in air at $450^{\circ} \mathrm{C}$ for $3 \mathrm{~h}$. Also, the HRTEM image is in accord with the estimate of $\sim 1.27 \mathrm{~nm}$ as the oxide scale determined from the conductivity measurements.

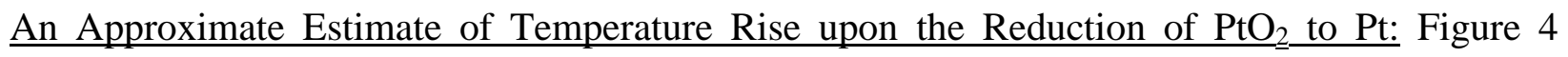
shows that when the atmosphere in the chamber was changed from vacuum to $10 \% \mathrm{H}_{2}+\mathrm{N}_{2}$, the temperature rose by about 10 degrees. This was attributed to the reaction of $\mathrm{PtO}_{2}$ with $\mathrm{H}_{2}$, which 
has an enthalpy of $-390 \mathrm{~kJ} \cdot \mathrm{mol}^{-1}$ [10]. Here we give a crude estimate of the expected temperature rise. Based on the estimated oxide scale thickness of $1.27 \mathrm{~nm}$ at the end of the carbon burn off, one can estimate the amount of $\mathrm{PtO}_{2}$ formed and thus the amount of heat released upon the reduction of $\mathrm{PtO}_{2}$ to $\mathrm{Pt}$ by reacting with $\mathrm{H}_{2}$. The total volume of the $\mathrm{PtO}_{2}$ formed in the nanoporous film during carbon burn off is given by

$$
V_{P t O_{2}}=A \ell S_{v} \delta(t)=2.79 \times 10^{-5} \mathrm{~cm}^{3}
$$

From the molar volume of $\mathrm{PtO}_{2}$ and the enthalpy of the reaction, the estimated value of the heat released is $0.49 \mathrm{~J}$. The total volume of Pt in the film is estimated to be $v_{P t}=5.625 \times 10^{-5} \mathrm{~cm}^{3}$. Based on the known heat capacity of Pt, if all of the heat released were adiabatically contained in the nanoporous film, the rise in temperature would have been $\Delta T \sim 3,100$ degrees! However, heat transfer is expected to occur to the glass slide and to the other adjacent regions. Obviously, the mass of the glass slide is orders of magnitude greater than the mass of the nanoporous film. In addition, heat transfer will also occur to other parts of the sample, including the electrical leads. Even the mass of the thermocouple tip is much larger than the mass of the nanoporous film. Thus, the measured temperature rise of $\sim 10$ degrees is very reasonable. The present results also suggest that the temperature of the nanoporous films must have increased by much greater than the measured 10 degrees, with slow heat dissipation occurring from the film to the adjacent regions. It is thus possible that some microstructural changes likely occurred during the reduction of $\mathrm{PtO}_{2}$ to Pt.

Comparison with Related Experiments on PEMFC: Proton exchange membrane fuel cells (PEMFC) with nanosize Pt supported on carbon as both cathode and anode catalysts are typically operated at $80^{\circ} \mathrm{C}$. It is well known that catalyst degradation primarily occurs at the cathode and 
especially at high electric potentials [2-6]. At high potentials, such as at open circuit voltage (OCV) conditions, the oxidation of $\mathrm{Pt}\left(\mathrm{Pt} \rightarrow \mathrm{Pt}^{2+}\right)$ is favored. Thus, greater cathode degradation occurs under open circuit conditions than when the fuel cell is under load. Ionized platinum is subject to dissolution and also leads to particle growth and thus loss of catalytic activity [15]. Most of the studies on the oxidation of Pt related to PEMFC (and other types of fuel cells and electrochemical devices) are conducted using cyclic voltammetry (CV). In cyclic voltammograms, a platinum oxidation peak is observed at $\sim 0.82 \mathrm{~V}$. It is to be noted that in cyclic voltammetry, an external voltage is applied. In the present work, no external voltage is applied. Thus, oxidative conditions the samples were exposed to correspond to open circuit conditions of a typical PEMFC.

Extensive studies have been conducted in aqueous media to investigate the oxidation of platinum. Yet, direct observation of an oxide scale under typical operating conditions of a PEMFC has been elusive. Extremely slow growth of the oxide scale, as determined in the present work, and the dissolution of $\mathrm{Pt}$ as $\mathrm{Pt}^{2+}$ in an aqueous medium of PEMFC (and either reprecipitation to cause Ostwald ripening and/or transport into the membrane), makes it practically impossible to conclusively demonstrate the formation of platinum oxides in PEMFC catalysts, that can be confirmed by some sort of direct (e.g. microstructural) evidence. The present approach of using nanoporous Pt films in an oxygen containing atmosphere (in the absence of water and without any applied voltage) allows for the experimental determination of the kinetic parameters $\left(k_{\text {eff }}\right.$ and $\left.D_{\text {eff }}\right)$ that describe Pt oxidation. Also, the estimated parameters $\left(k_{\text {eff }}\right.$ and $D_{\text {eff }}$ ) clearly demonstrate that it is not possible to develop a measurable oxide scale thickness within the time frame of a typical experiment or even during the lifetime of a typical PEMFC 
stack. Even though the oxide scale formed at $80^{\circ} \mathrm{C}$ is sub-monolayer, the validity of the approach was confirmed by the carbon burn off experiment at $450^{\circ} \mathrm{C}$ in air. If one assumes that the lowest possible observable oxide thickness is $\sim 0.5 \mathrm{~nm}$ (e.g. by microscopy), based on the parameters measured in the present work, the required time to form a $0.5 \mathrm{~nm}$ oxide scale at $80^{\circ} \mathrm{C}$ will be > 80 years! This also suggests that a large decrease in activity for the oxygen reduction reaction (ORR), such as would be expected if a continuous oxide scale is formed, is not expected to occur in PEMFC. The only option to obtain microscopic evidence of oxide film formation is therefore to increase the temperature and conduct oxidation experiments for a sufficiently long period of time. An exceptionally low value of the estimated $D_{\text {eff }}$ at $80^{\circ} \mathrm{C}$ explains why in none of the reported studies the formation of an oxide scale at the operating temperature of a PEMFC has been demonstrated [16]. On the other hand, the observation in the present work that the electrical resistance increases when a nanoporous Pt film is exposed to air and that XPS spectra confirm the presence of surface oxygen, clearly demonstrate that oxygen does readily interact with the surface of Pt. This interaction may be chemisorption of oxygen.

Other techniques that have been used to investigate the interaction of oxygen with Pt surfaces include the electrochemical quartz microbalance (EQCM) technique [17]. It was deduced that up to 0.47 monolayer of oxygen could be formed under an applied voltage of up to $1.2 \mathrm{~V}$. However, when no voltage was applied, the oxygen coverage was about 2 orders of magnitude lower. While many uncertainties exist in the interpretation of EQCM data due to the presence of water and issues related to the interpretation of data, very low coverage in the absence of an applied voltage is in accord with the present estimate of $\sim 2.55 \times 10^{-10} \mathrm{~cm}$ as the film thickness at $80^{\circ} \mathrm{C}$. It is to be noted that EQCM technique does not yield a direct confirmation of the possible existence 
of an oxide scale and can only be regarded as possibly providing an indirect evidence of oxidation.

One of the possible mechanisms of oxygen interaction with Pt in a PEMFC electrode (or other electrochemical devices with $\mathrm{Pt}$ as the electrode) is the so-called place exchange reaction in which $\mathrm{O}$ atoms can enter the Pt lattice [18-21]. It is possible that some of the $\mathrm{O}$ may enter the lattice. However, the possible change in electrical conductivity of Pt with place-exchanged $\mathrm{O}$ may be small (assuming it forms a dilute solution of $\mathrm{O}$ in $\mathrm{Pt}$ ). Thus, the present experiments would likely not be able to provide evidence of this mechanism of oxygen incorporation. It is also to be mentioned that unequivocal evidence of the occurrence of the place exchange mechanism (in which surface $\mathrm{O}$ and interior Pt atoms can exchange places) has yet not been presented.

Other Models for the Kinetics of Oxidation: Several authors have investigated the kinetics of oxidation of metals in electrochemical systems as well as in gaseous atmospheres. The model discussed here is based on the well-known parabolic law of oxidation by Wagner, which is based on the thermodynamics of oxidation and the kinetics of transport [13]. It is well known that the kinetics of oxidation of numerous metals and alloys can be described by the Wagner model. In many electrochemical systems, one of the most commonly used models is the logarithmic model, such as that described by Conway et al. [22]. Many of these studies have used the logarithmic model described by Cabrera and Mott [23]. The origin of the logarithmic (or inverse logarithmic) models lies in the experimental observation and that often the oxide scale thickness varies nearly linearly with logarithm of time. Such a model, however, is empirical and does not afford the 
incorporation of fundamental thermodynamic and or kinetic parameters in the manner that the Wagner model does. Thus, in the present work, the Wagner model based on the occurrence of interfacial reactions at gas/solid and solid/solid interfaces, and diffusion through the oxide scale, was used to interpret the experimental observations. The measured parameters in the present work can be clearly related to the thermodynamics of oxidation and the kinetics of transport.

Experimental studies on the interaction of gaseous species with metallic surfaces (chemisorption and/or oxidation) are important in many chemical and catalytic processes. Very sluggish reactions and transport at low temperatures (temperatures which are often of interest from a technological standpoint) make it difficult to study them experimentally. The present work shows that one approach is to study them is by using nanoporous samples with a very high surface area per unit volume. This enhances the kinetics of the reactions (because of the effect of geometry), making it possible to determine the requisite kinetic parameters within a reasonable time frame.

\section{Summary}

Nanoporous Pt films were fabricated on glass sides by co-sputtering Pt and carbon, and subsequently burning off the carbon. The kinetics of oxidation were investigated at $80^{\circ} \mathrm{C}$ in air by measuring the electrical resistance of the film as a function of time. The increase in resistance was described by a model which includes two interfacial steps and one diffusive step. The model is essentially the Wagner model of oxidation. From the analysis of resistance vs. time data, an

effective diffusion coefficient, $D_{\text {eff }}$, and an effective interface reaction parameter, $k_{e f f}$, were 
estimated. At $80^{\circ} \mathrm{C}$, the estimated value of $D_{e f f}$ is $\sim 1 \times 10^{-24} \mathrm{~cm}^{2} \mathrm{~s}^{-1}$. The very low value of $D_{e f f}$ suggests that over the duration of the experiments, the oxide scale formed was sub-monolayer, and thus was not continuous. Many studies on the oxidation of Pt in PEMFC and liquid media have also concluded that the estimated oxide scale is sub-monolayer. In this context, the present work which examined Pt oxidation in a gaseous phase under no applied voltage and most of the literature which has investigated oxidation in aqueous media (typically using cyclic voltammetry), come to similar conclusions. While numerous studies have shown indirect evidence of oxygen adsorption/oxide scale formation, the present results suggest that a continuous film of Pt-oxide is not expected to form at the typical PEMFC operating temperature due to very slow kinetics. These expectations are supported by the available literature, which also shows that a directly measurable (e.g. by microscopy) continuous oxide scale does not form under the typical PEMFC operating conditions (temperature, time, and operating voltage). Extrapolation of the data suggests that a contiguous film should form at higher temperatures and will require longer times of oxidation. At $450^{\circ} \mathrm{C}$, the estimated oxide scale thickness is $\sim 1.27 \mathrm{~nm}$ in $3 \mathrm{~h}$ and the corresponding $D_{\text {eff }}$ is $\sim 1.495 \times 10^{-18} \mathrm{~cm}^{2} \mathrm{~s}^{-1}$. HRTEM images are indeed consistent with the formation of a 1 to $2 \mathrm{~nm}$ oxide scale upon oxidation in air at $450^{\circ} \mathrm{C}$ for $3 \mathrm{~h}$. The present work shows that a study of electrical resistance of nanoporous films as a function of time affords a method for investigating the kinetics of oxidation at rather low temperatures in a reasonable time.

Acknowledgements: Work of Liangzhu Zhu (experimental design and measurements, and analysis of data), and Anil Virkar (analysis and modeling) was supported by DOE EFRC under 
Grant DE-SC0001061 as a flow-through from the University of South Carolina. Work of Siddarth Kapoor (sample fabrication and measurements) and Quintin Parry (sample fabrication and measurements) was supported by NSF under Grant Number CBET 0931080. Work of Ajay Nahata (design of experiments and analysis of data) was supported by NSF MRSEC under Grant Number DMR 11-21252. The authors are grateful to Lei Zhang, Brian Baker, Brian Denever, Randy Polson and David Belnap, respectively, for discussions regarding experimental procedure, and assistance with sample preparation, and the use of XPS, FIB and HRTEM facilities. 


\section{References}

[1] H. A. Gasteiger, S. S. Kocha, B. Sompalli, F. T. Wagner, Applied Catalysis B: Environmental, 56 [1-2] 9-35 (2005).

[2] X. Yu and S. Ye, J. Power Sources 172 145-154 (2007).

[3] Y. Shao, G. Yin and Y. Gao, J. Power Sources, 171 558-566 (2007).

[4] J. Xie, J. L. Wood, K. L. More, P. Atanassov, R. L. Borup, J. Electrochem. Soc., 152 A1011 (2005).

[5] P. J. Ferreira, G. J. La, O. Y. Shao-Horn, D. Morgan, R. Makharia, S. Kocha, H. A. Gasteiger, J. Electrochem. Soc., 152 A2256 (2005).

[6] W. Bi, Q. Sun, Y. Deng, T. F. Fuller, Electrochimica Acta, 54 1826-1833 (2009).

[7] R. J. Berry, Surface Science, 76 415-442 (1978).

[8] E. A. Underwood, 'Quantitative Stereology’, Addison-Wesley Interscience (1970). 
[9] E. L. Cussler, 'Diffusion: Mass transfer in fluid systems', Cambridge University Press, (1995).

[10] Y. Nagano, Journal of Thermal Analysis and Calorimetry, 69, 831-839 (2002).

[11] I. Barin, 'Thermochemical Data of Pure Substances: Parts I \& II', VCH Publication, Weinheim, Germany (1993).

[12] Y. Abe, M. Kawamura, K. Sasaki, Jpn. J. Appl. Phys., Vol. 38 No. 4A, 2092-2096 (1999).

[13] P. Kofstad, 'High-temperature oxidation of metals', J. Wiley publication, New York (1966).

[14] Defect and Diffusion Forum, Diffusion in Ceramics - 10 Years of Research, Editor: D. J. Fisher, Volume 169-170, Scitec Publications (1999).

[15] R. M. Darling, J. P. Meyers, J. Electrochem. Soc., 150 A1523 (2003).

[16] E. L. Redmund, B. P. Setzler, F. M. Alamgir, T. F. Fuller, Phys. Chem. Chem. Phys., 16 $5301-5311(2014)$.

[17] A. Kongkanand, J. M. Ziegelbauer, J. Phys. Chem. C, 116 3684-3693 (2012).

[18] D. C. Johnson, D. T. Napp, S. Buckenst, Electrochim. Acta, 15 1493-1509 (1970). 
[19] A. Teliska, W. E. O’Grady, D. E. Ramaker, J. Phys. Chem. B, 109 8076-8084 (2005).

[20] H. Angerstein-Kozlowska, B. E. Conway, W. B. A. Sharp, J. Electroanal. Chem., 439 (1973).

[21] B. E. Conway, Prog. Surf. Sci., 161 (1984).

[22] B. E. Conway, B. Barnett, H. Angerstin-Kozlowska, B. V. Tilak, J. Chem. Phys. 93 (11) $8361-8373(1990)$.

[23] N. Cabrera, N. F. Mott, Rep. Prog. Phys. 12163 (1949). 
Oxidation/Reduction Studies on Nanoporous Platinum Films by Electrical Resistance

\title{
Measurements
}

Liangzhu Zhu ${ }^{1}$, Siddharth Kapoor ${ }^{2}$, Quintin Parry ${ }^{1}$, Ajay Nahata ${ }^{2}$, and Anil V. Virkar ${ }^{1, *}$

\author{
${ }^{1}$ Department of Materials Science and Engineering \\ ${ }^{2}$ Department of Electrical and Computer Engineering \\ University of Utah
}

Salt Lake City, UT 84112

*Contributing Author

Email: anil.virkar@utah.edu 


\section{Figure Captions}

Figure 1: (a) A schematic diagram of the test set-up. (b) A photograph of the test set-up.

Figure 2: An SEM image of a nanoporous Pt film deposited on a glass slide.

Figure 3: Temperature and electrical resistance of the film while heating in vacuum and after temperature stabilization. Temperature oscillations occur due to the on/off nature of the power controller and relatively low thermal inertia of the sample and the surroundings in vacuum.

Figure 4: Temperature and electrical resistance after the introduction of a gaseous mixture containing $10 \% \mathrm{H}_{2}+\mathrm{N}_{2}$ followed again by turning on the vacuum pump.

Figure 5: Plots of temperature and electrical resistance vs. time after the introduction of air into the chamber.

Figure 6: XPS spectra from the Pt film. (a) As-deposited Pt $+\mathrm{C}$ film. (b) After burning off carbon in air at $450^{\circ} \mathrm{C}$ for $3 \mathrm{~h}$. (c) After exposure to $10 \% \mathrm{H}_{2}+\mathrm{N}_{2}$ atmosphere at $80^{\circ} \mathrm{C}$. (d) After exposure to air at $80^{\circ} \mathrm{C}$ for $18 \mathrm{~h}$.

Figure 7: A schematic of the nanoporous Pt film with a thin platinum oxide layer on the internal surfaces of the nanostructured film. 
Figure 8: A schematic of a $\mathrm{PtO}_{2}$ film on $\mathrm{Pt}$ and the variation of the chemical potential of oxygen, $\mu_{O_{2}}$, through the film.

Figure 9: A plot of $\frac{1}{R(t)}$ vs. $t$ as $t \rightarrow 0$. The intercept is $0.0367 \Omega^{-1}$ and the slope is $-3.01 \times 10^{-6}$ $\Omega^{-1} \mathrm{~S}^{-1}$

Figure 10: A plot of $\frac{1}{R(t)}$ vs. $\sqrt{t}$ at long times. The intercept is $0.0335 \Omega^{-1}$ and the slope is -3.98 $\mathrm{X} 10^{-6} \Omega^{-1} \mathrm{~s}^{-1 / 2}$.

Figure 11: A HRTEM image of the sample after carbon burn off in air at $450^{\circ} \mathrm{C}$ for $3 \mathrm{~h}$. The grain (particle) size is $\sim 5 \mathrm{~nm}$. The central light region is a pore. The insets show (a and b) the FIB preparation of sample for HRTEM analysis, and 'c' shows the Fast Fourier transform (FFT) pattern of the whole image representing f.c.c. crystals along the $<110>$ zone axis.

Figure 12: A higher magnification image of the region surrounding the pore in Figure 11. The regions marked as c, d, and e, exhibit a different morphology than the interior regions. Insets are FFT patterns corresponding to regions a and $b$, and the inverse FFT images which clearly show Pt lattice image corresponding to $\mathrm{Pt}\{111\}$ planes. The marked regions c, d, and e are identified as the oxide scale. The thickness of the oxide scale is about 1 to $2 \mathrm{~nm}$. 

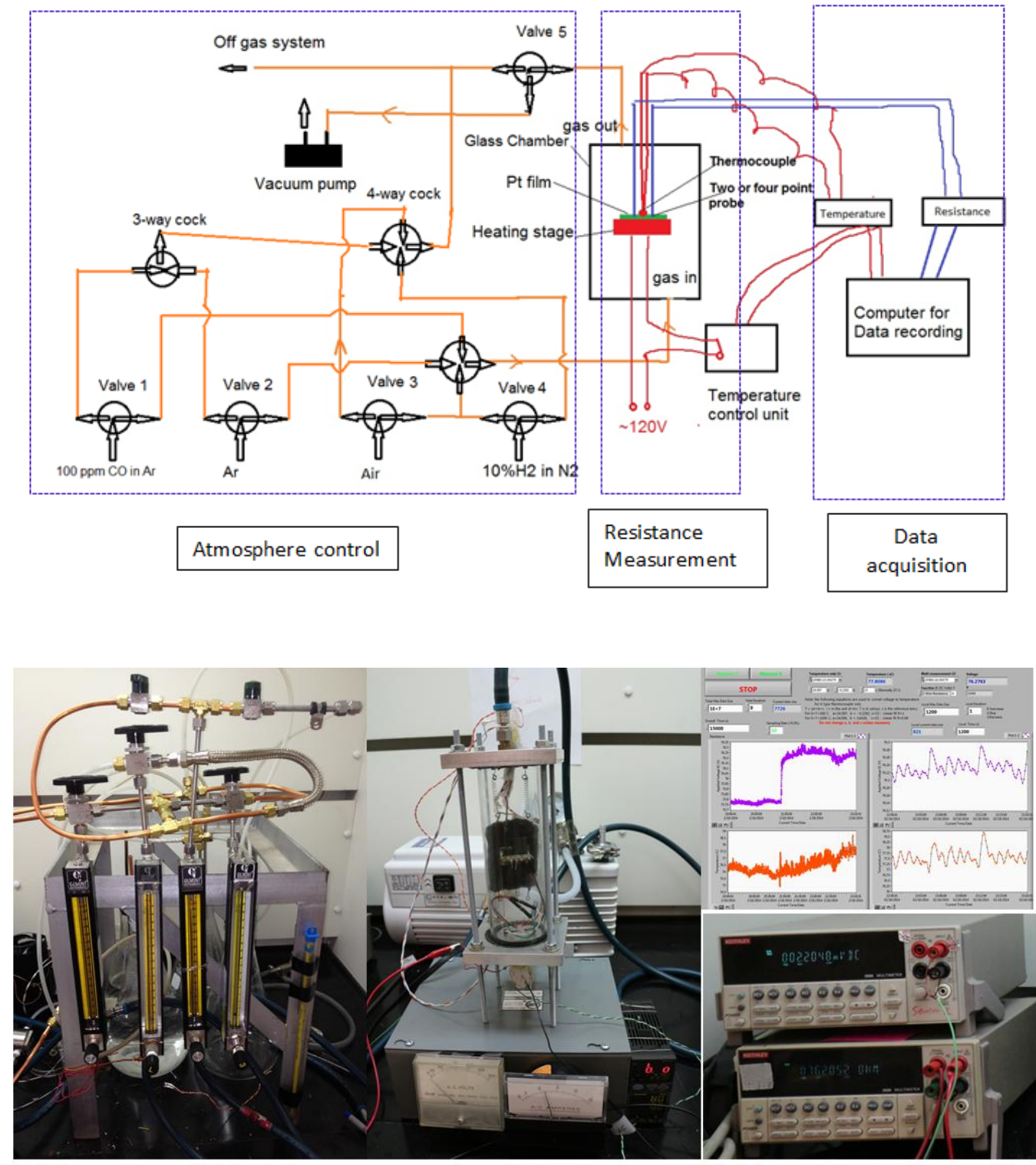

Atmosphere control

Resistance measurement

Data acquisition

Figure 1: (a) A schematic diagram of the test set-up. (b) A photograph of the test set-up. 


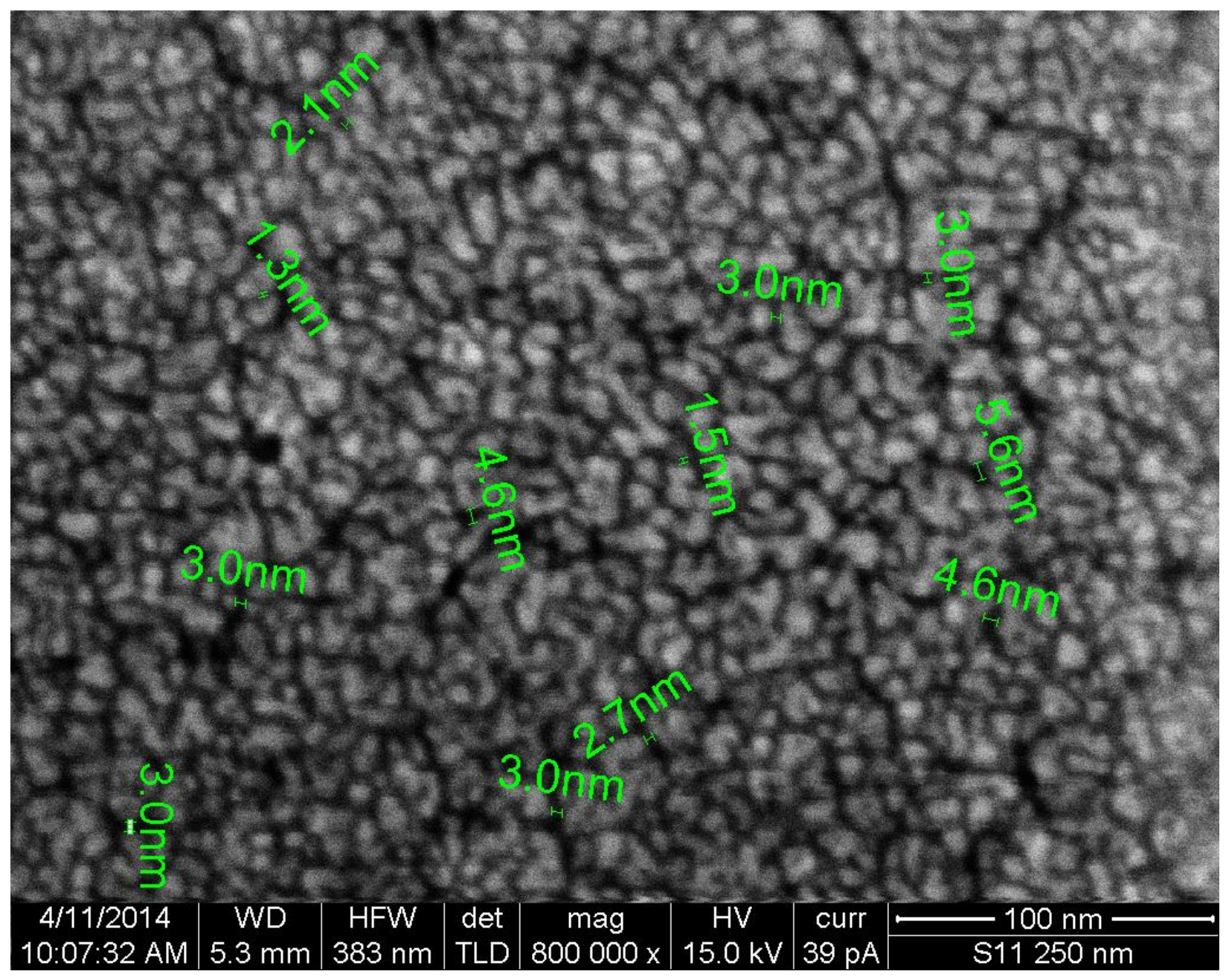

Figure 2: An SEM image of a nanoporous Pt film deposited on a glass slide. 


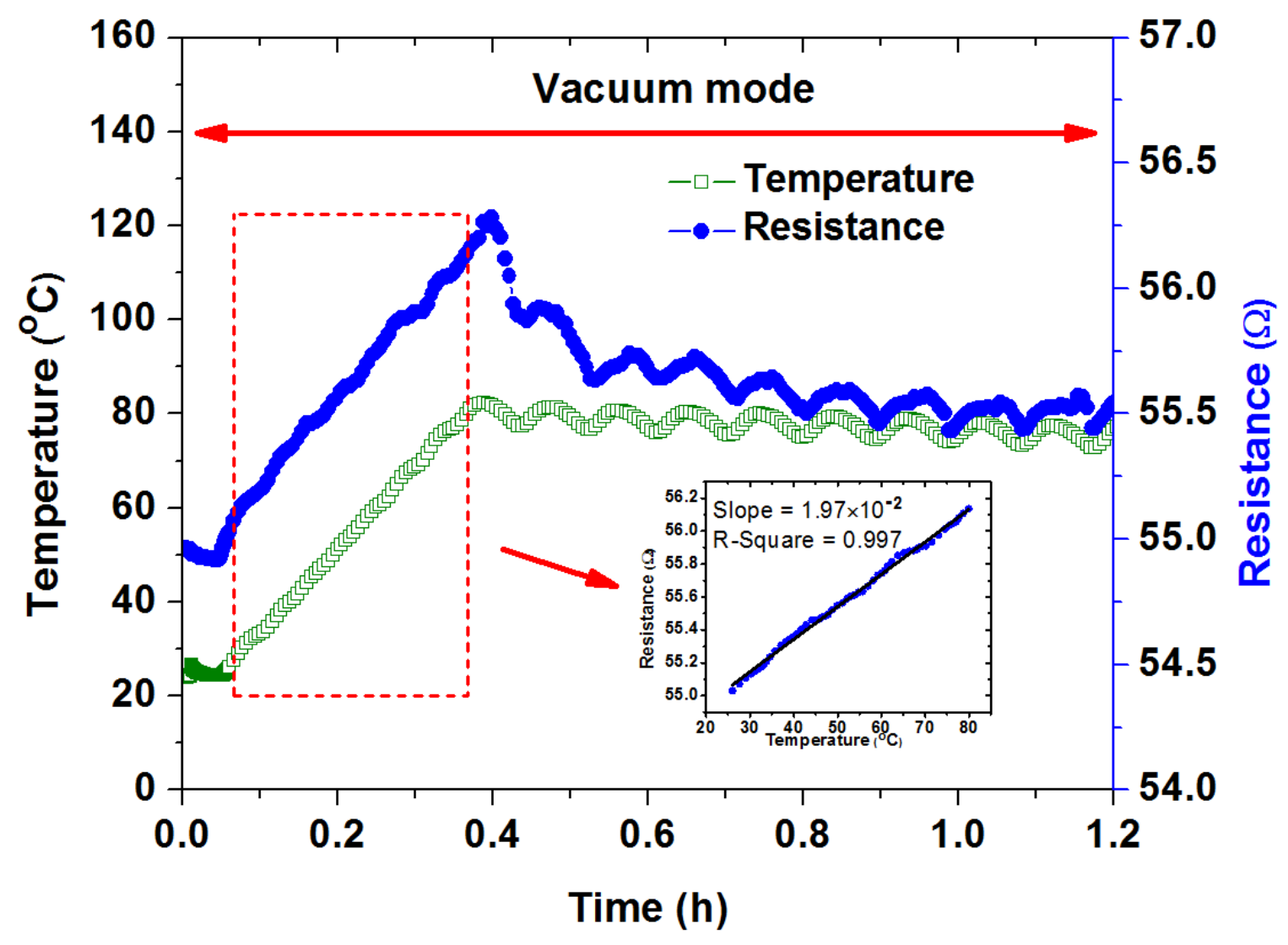

Figure 3: Temperature and electrical resistance of the film while heating in vacuum and after temperature stabilization. Temperature oscillations occur due to the on/off nature of the power controller and relatively low thermal inertia of the sample and the surroundings in vacuum. 


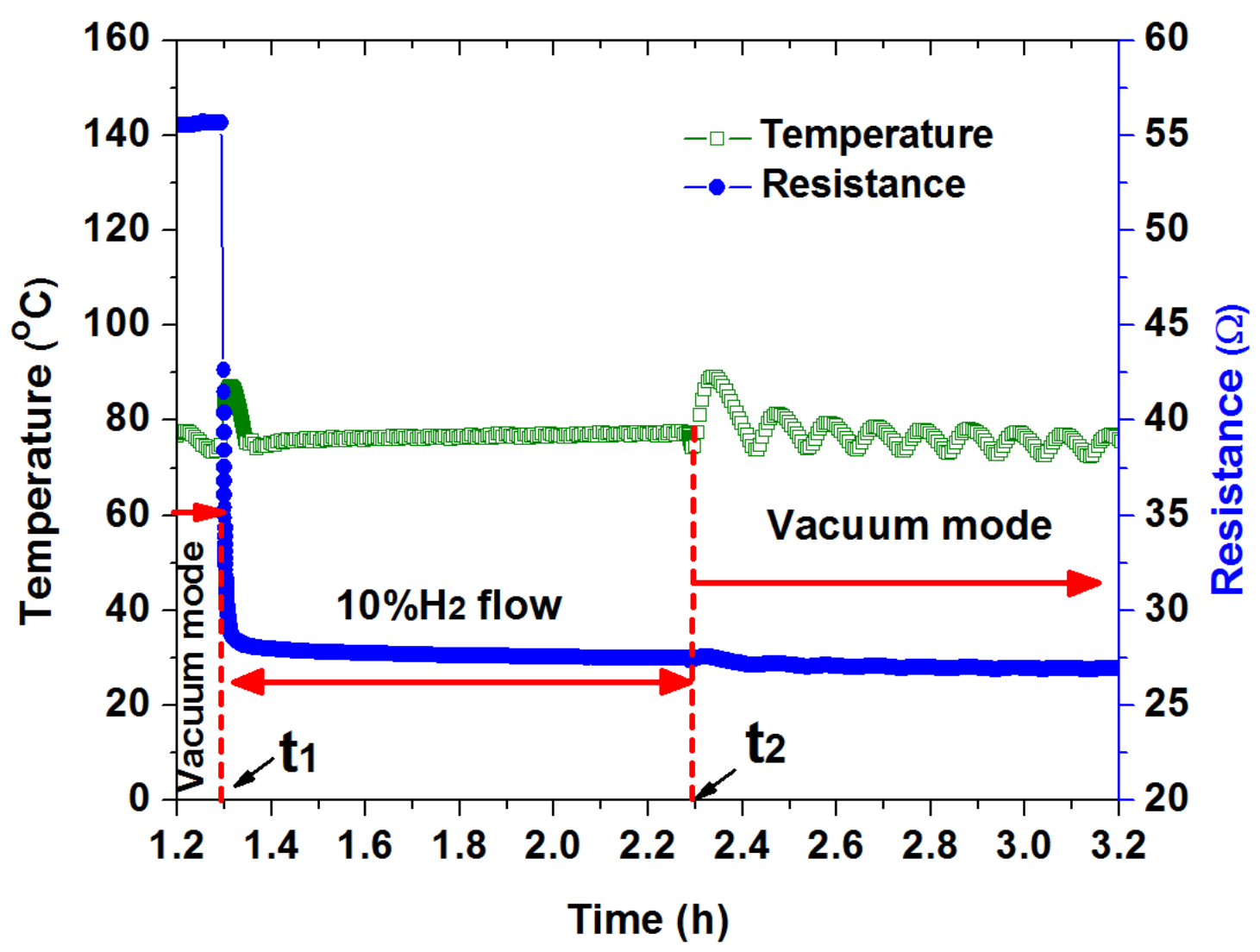

Figure 4: Temperature and electrical resistance after the introduction of a gaseous mixture containing $10 \% \mathrm{H}_{2}+\mathrm{N}_{2}$ followed again by turning on the vacuum pump. 


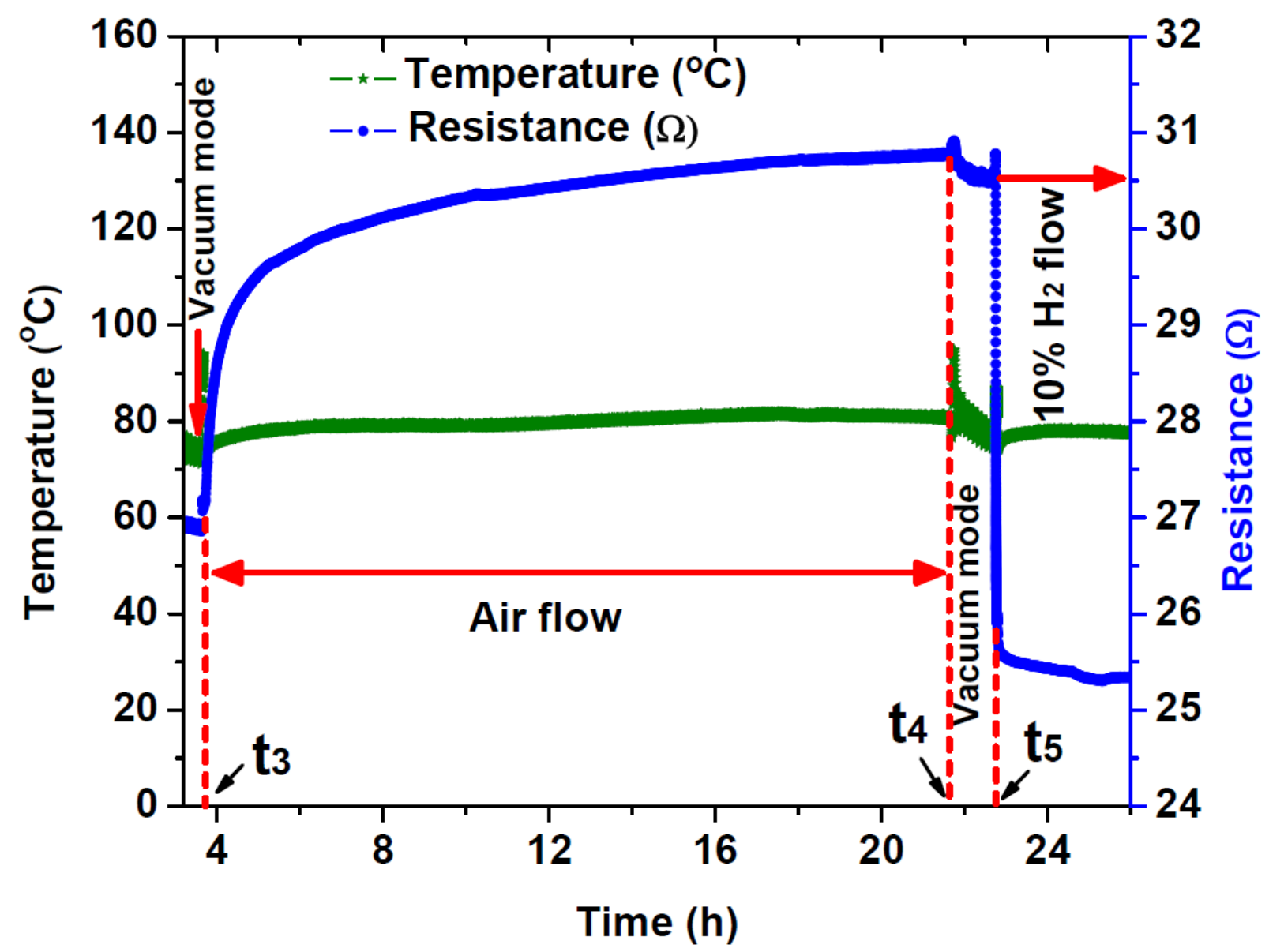

Figure 5: Plots of temperature and electrical resistance vs. time after the introduction of air into the chamber. 


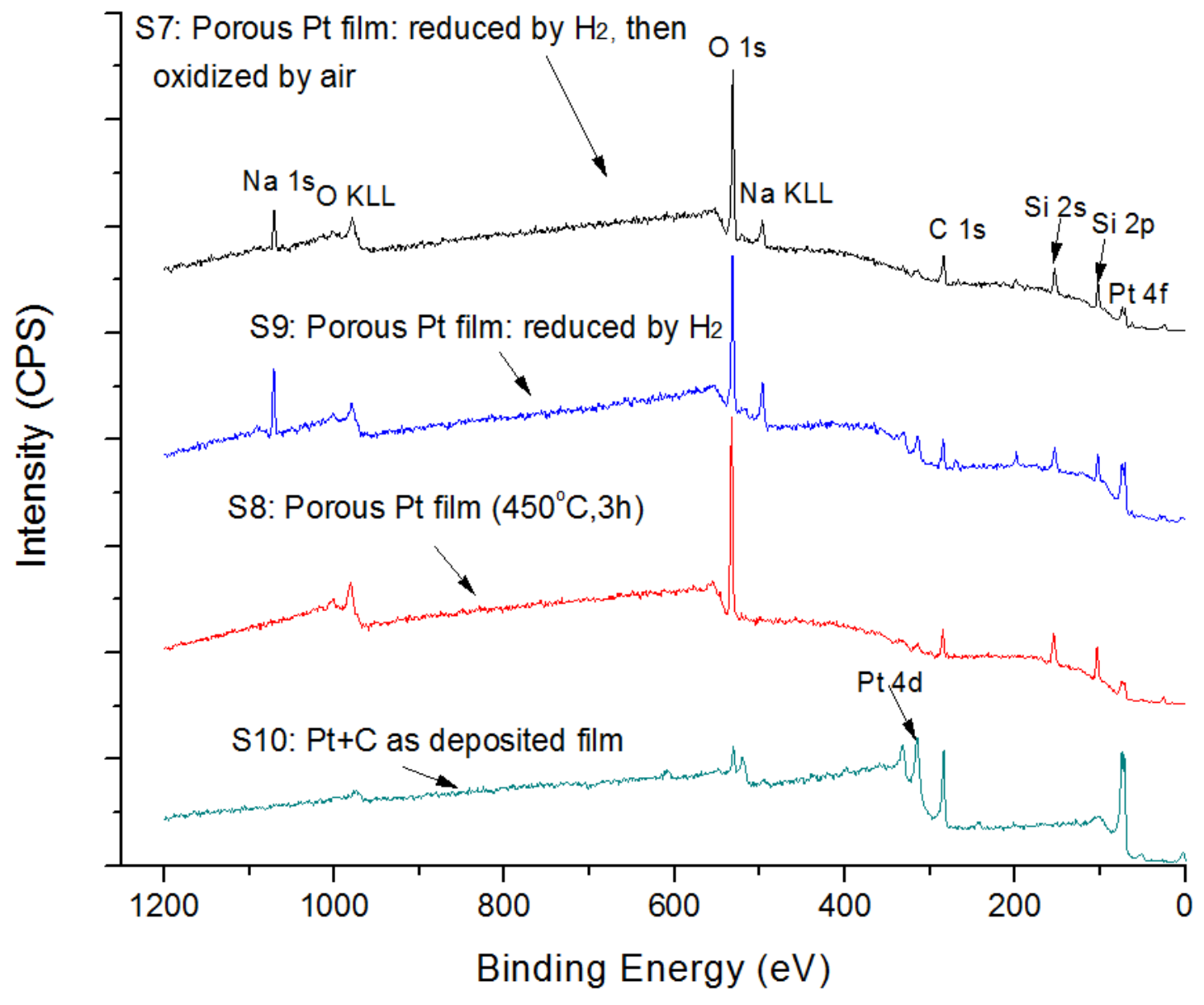

Figure 6: XPS spectra from the Pt film. (a) As-deposited Pt $+\mathrm{C}$ film. (b) After burning off carbon in air at $450^{\circ} \mathrm{C}$ for $3 \mathrm{~h}$. (c) After exposure to $10 \% \mathrm{H}_{2}+\mathrm{N}_{2}$ atmosphere at $80^{\circ} \mathrm{C}$. (d) After exposure to air at $80^{\circ} \mathrm{C}$ for $18 \mathrm{~h}$. 


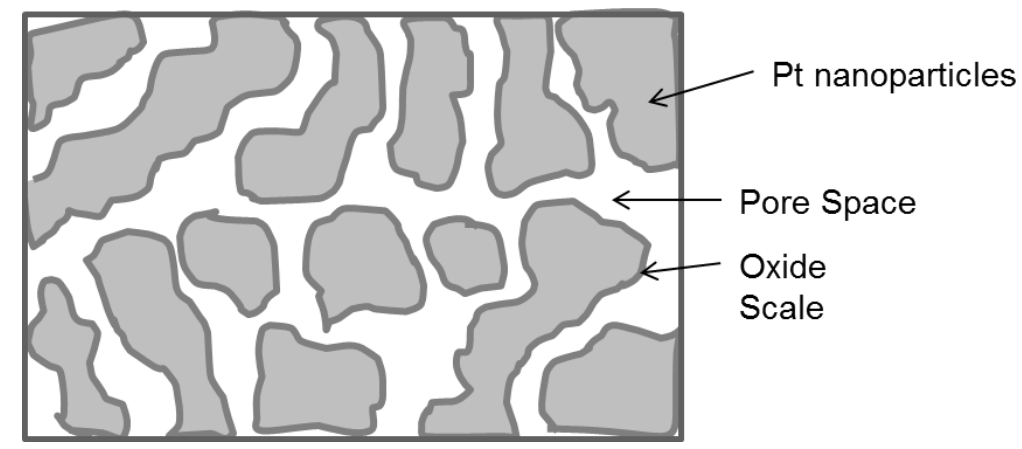

Figure 7: A schematic of the nanoporous Pt film with a thin platinum oxide layer on the internal surfaces of the nanostructured film. 


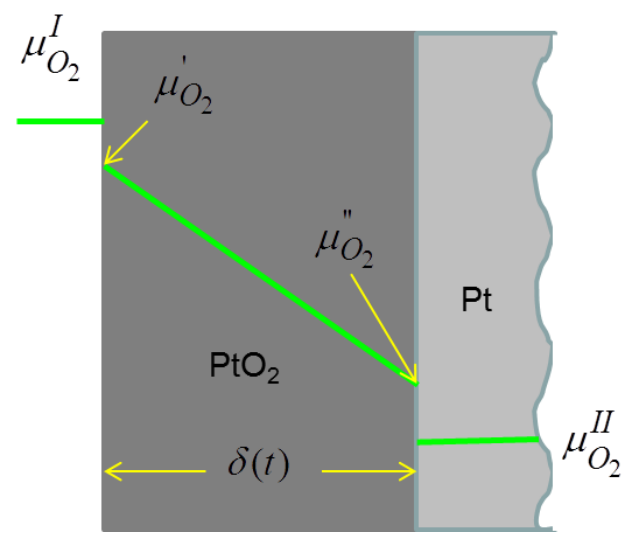

Figure 8: A schematic of a $\mathrm{PtO}_{2}$ film on $\mathrm{Pt}$ and the variation of the chemical potential of oxygen, $\mu_{O_{2}}$, through the film. 


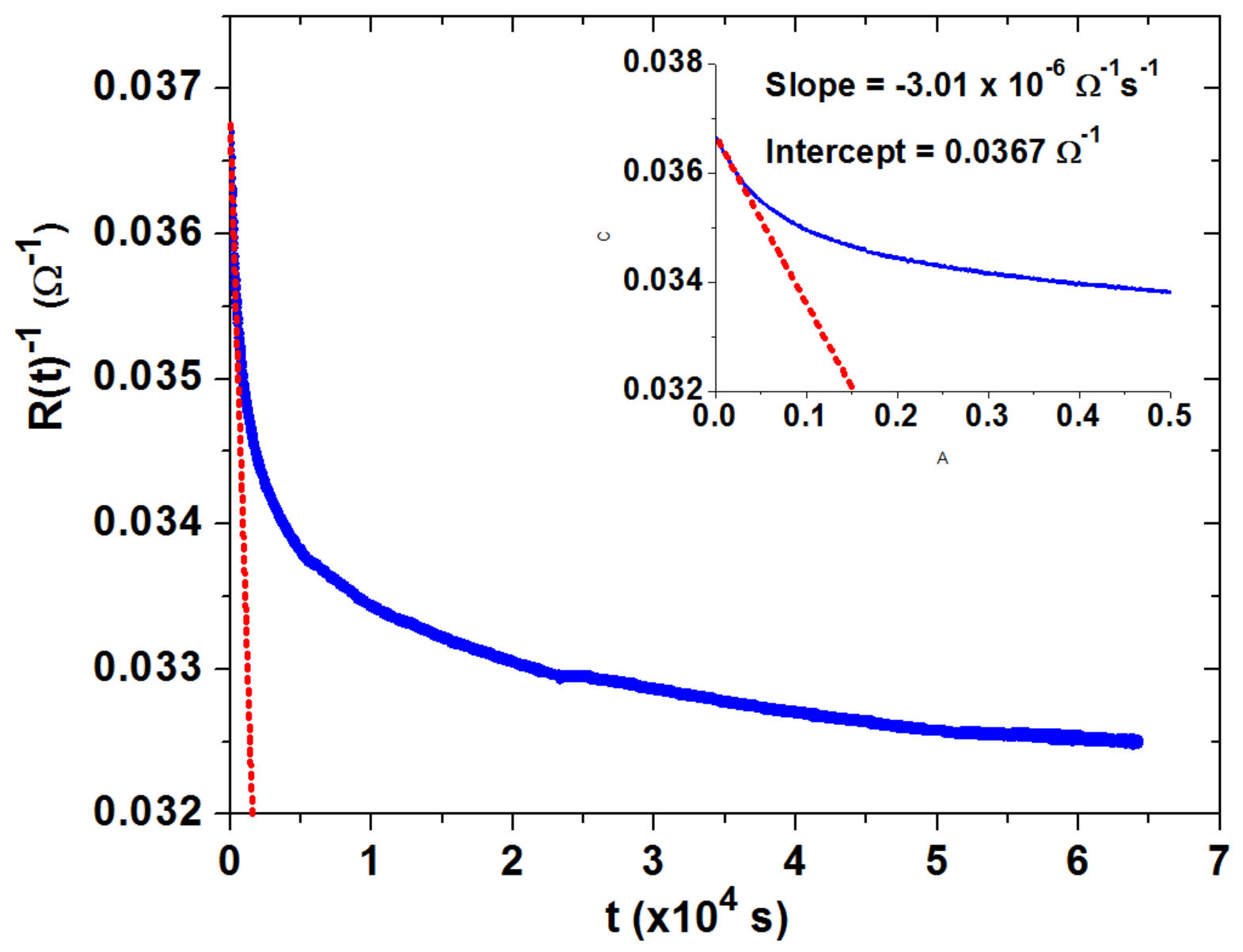

Figure 9: A plot of $\frac{1}{R(t)}$ vs. $t$ as $t \rightarrow 0$. The intercept is $0.0367 \Omega^{-1}$ and the slope is $-3.01 \times 10^{-6}$ $\Omega^{-1} \mathrm{~s}^{-1}$. 


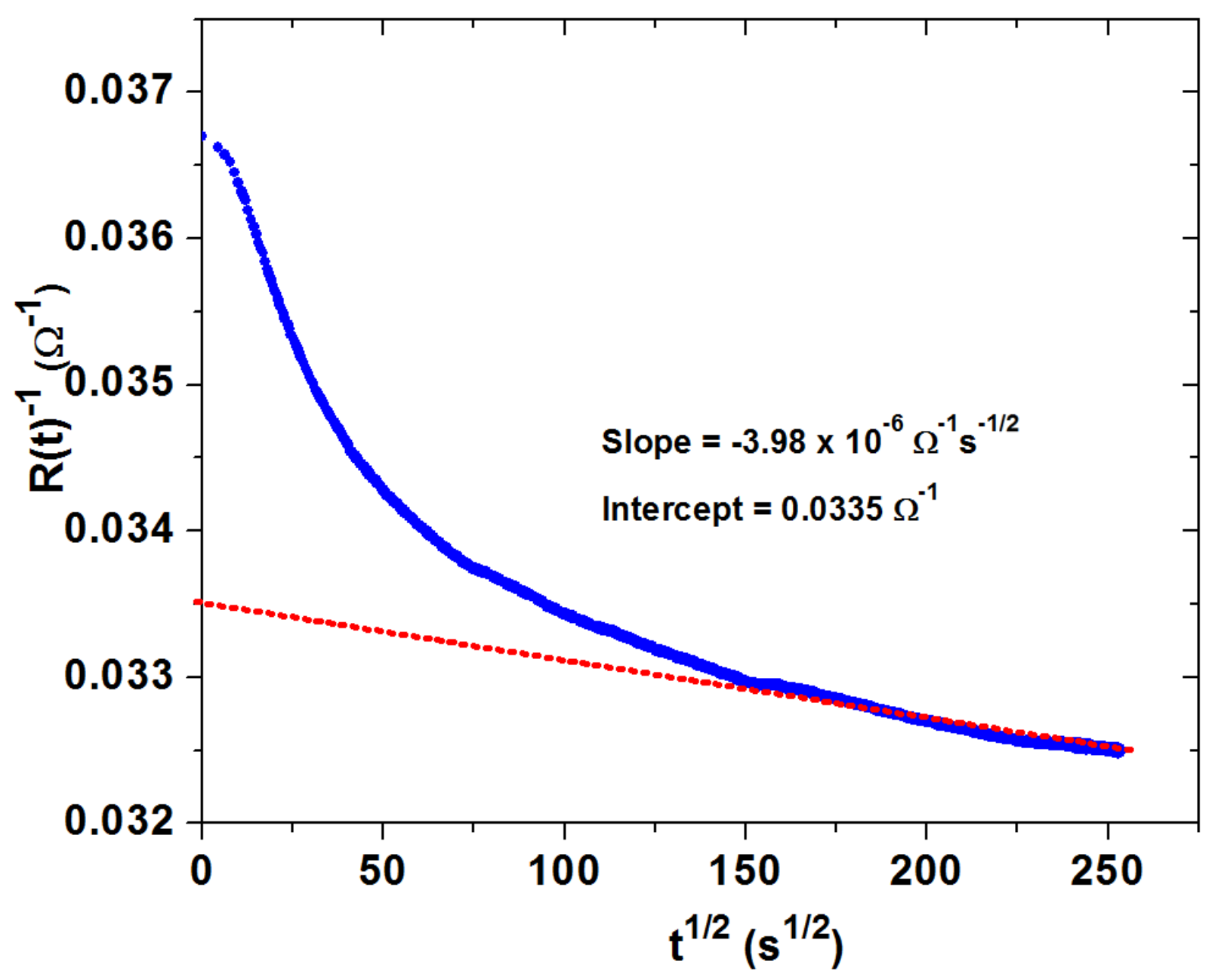

Figure 10: A plot of $\frac{1}{R(t)}$ vs. $\sqrt{t}$ at long times. The intercept is $0.0335 \Omega^{-1}$ and the slope is -3.98 $\mathrm{x} 10^{-6} \Omega^{-1} \mathrm{~s}^{-1 / 2}$. 


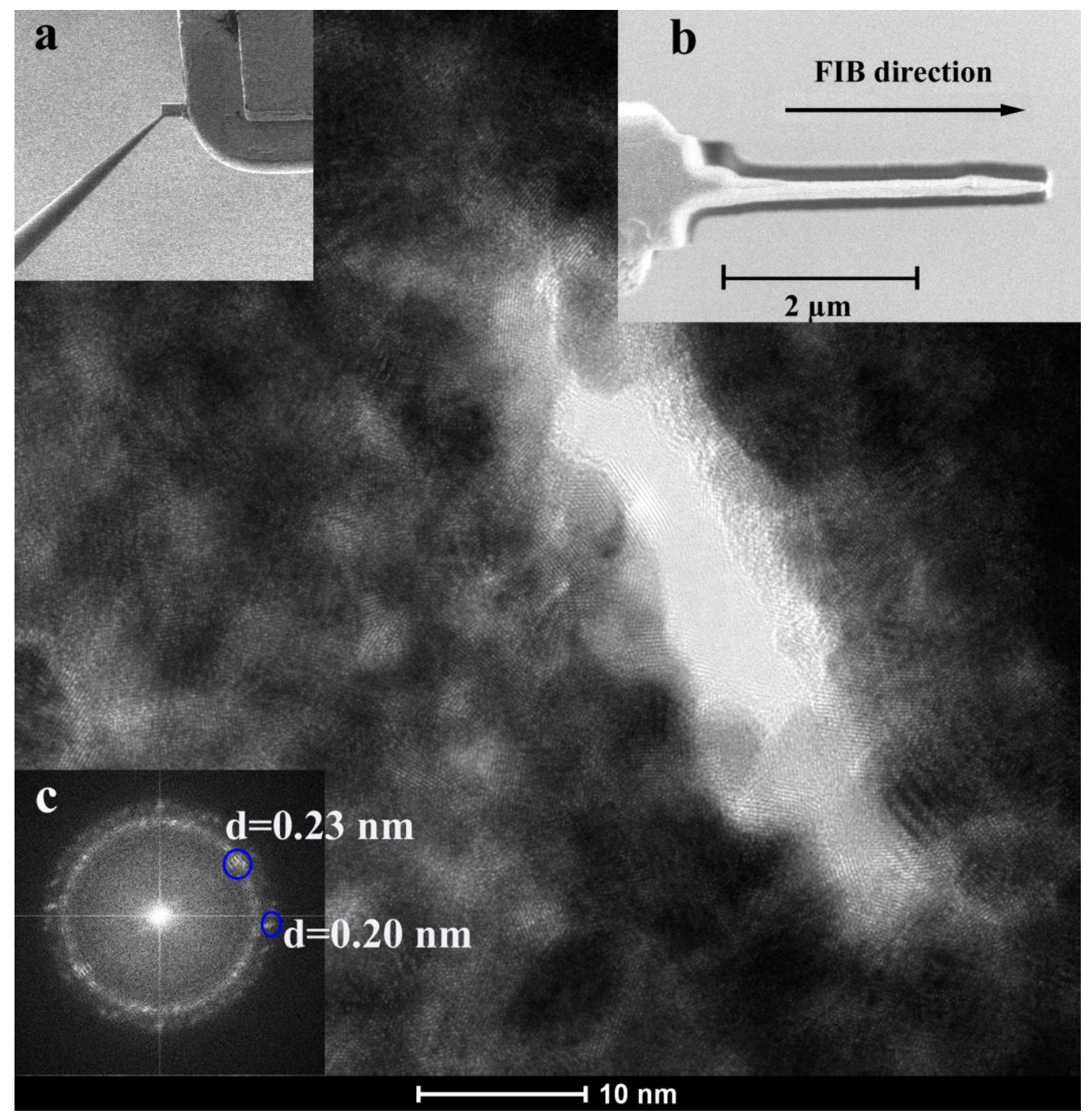

Figure 11: A HRTEM image of the sample after carbon burn off in air at $450^{\circ} \mathrm{C}$ for $3 \mathrm{~h}$. The grain (particle) size is $\sim 5 \mathrm{~nm}$. The central light region is a pore. The insets show (a and b) the FIB preparation of sample for HRTEM analysis, and 'c' shows the Fast Fourier transform (FFT) pattern of the whole image representing f.c.c. crystals along the $<110>$ zone axis. 


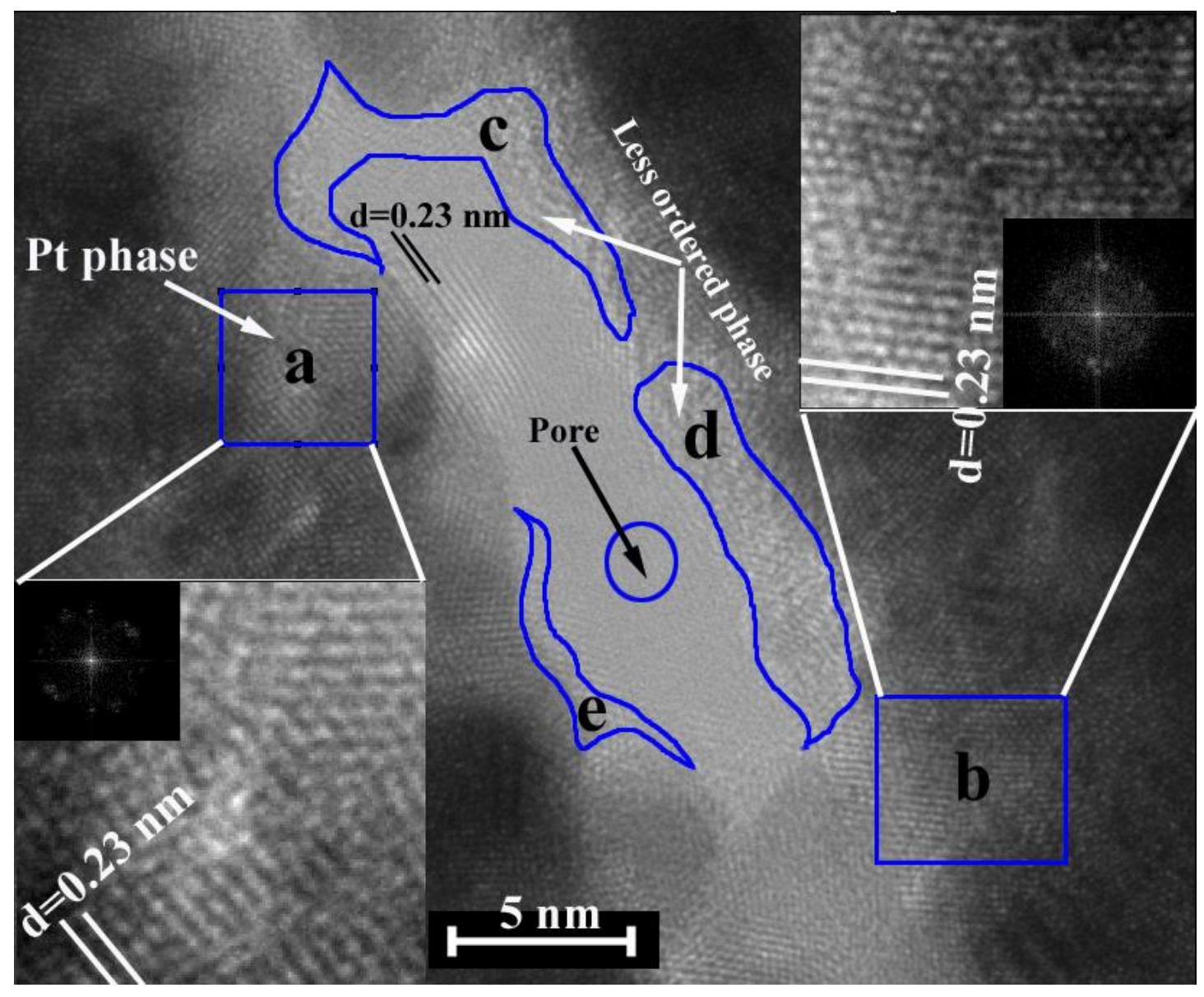

Figure 12: A higher magnification image of the region surrounding the pore in Figure 11. The regions marked as $\mathrm{c}, \mathrm{d}$, and e, exhibit a different morphology than the interior regions. Insets are FFT patterns corresponding to regions a and b, and the inverse FFT images which clearly show Pt lattice image corresponding to $\mathrm{Pt}\{111\}$ planes. The marked regions $\mathrm{c}, \mathrm{d}$, and e are identified as the oxide scale. The thickness of the oxide scale is about 1 to $2 \mathrm{~nm}$. 